\title{
Novel Fuzzy and Game Theory based Clustering and Decision Making for VANETs
}

\author{
Ayoub Alsarhan, Yousef Kilani, Ahmed Al-Dubai, Senior, IEEE, Albert Y. Zomaya, Fellow, \\ IEEE, and Amir Hussain, Senior, IEEE
}

\begin{abstract}
Different studies have recently emphasized the importance of deploying clustering schemes in Vehicular ad hoc Network (VANET) to overcome challenging problems related to scalability, frequent topology changes, scarcity of spectrum resources, maintaining clusters stability, and rational spectrum management. However, most of these studies addressed the clustering problem using conventional performance metrics while spectrum shortage, and the combination of spectrum trading and VANET architecture have not been tackled so far. Thus, this paper presents a new fuzzy logic based clustering control scheme to support scalability, enhance the stability of the network topology, motivate spectrum owners to share spectrum and provide efficient and cost-effective use of spectrum. Unlike existing studies, our context-aware scheme is based on multi-criteria decision making where fuzzy logic is adopted to rank the multi-attribute candidate nodes for optimizing the selection of cluster heads $(\mathrm{CH})$ s. Criteria related to each candidate node include: received signal strength, speed of vehicle, vehicle location, spectrum price, reachability, and stability of node. Our model performs efficiently, exhibits faster recovery in response to topology changes and enhances the network efficiency life time.
\end{abstract}

Index Terms - Clustering architecture, Cognitive network, Fuzzy logic, Multi-criteria decision making, Vehicular Ad-hoc Networks.

\section{INTRODUCTION}

$\mathrm{N}$ owadays, the rapid growth of the world population is indelibly tightly coupled with the massive growth in vehicular communications deployment and application. Recent advances in wireless communication technologies has led to the development of new communication paradigms where vehicles can communicate with each other, i.e. VANET, with the required infrastructure [1]. VANET supports a plethora of safety and non-safety related applications [1, 2]. However, these applications require low latency, and high-reliability communication at appropriate overall data rates.

Furthermore, a timely update of the network topology is a

- A. Alsarhan and Y. Kilani are with the Dept. of Computer Information Systems, Hashemite University, Jordan, E-mail: ayoubm@hu.edu.jo ; ymkilani@hu.edu.jo

- A. Al-Dubai and A. Hussain are with the School of Computing; Edinburgh Napier University, UK; E-mail: a.al-dubai@napier.ac.uk; a.hussain@napier.ac.uk

- A. Zomaya is with the School of Computer Science, University of Sydney, Australia E-mail: albert.zomaya@sydney.edu.au significant requirement for the operation of safety applications in this network. VANETs pose challenges related to their specific characteristics that distinguish them from other wireless networks. Beside the high mobility, the irregular distribution of nodes leads to frequent VANET partitions, particularly in highway scenarios [1-4]. Furthermore, the transmission range of vehicles is short, which reduces connectivity [1-4]. Hence, supporting dynamic data traffic in VANET cannot be guaranteed. Frequent network partitioning results in frequent intermittent connections that may lead to severe packet loss and significant latency [4].

In addition, VANETs are non-structured, autonomous, totally decentralized self-configured networks with distributed characteristics nodes [1-4]. These properties make resource management a challenging problem. Maintaining a global network topology in a centralized entity is essential in order to manage a large-scale VANET in a metropolitan area.

Indeed, VANET stability, reachability, and lifetime are among the most important challenges, facing the reliability, efficiency and feasibility of VANET, within growing arrays of applications and opportunities [3], [4]. As the topology changes rapidly and continuously, critical issues such as prolonging network lifetime and spectrum scarcity become a key element in maintaining predictable performance.

Although flat network topology has been accepted reasonably previously, there has been recently a dramatic increase to access the limited spectrum of VANETs. This has strained the effectiveness of the traditional flat network topology. Hence, a hierarchical network topology, termed cluster, has been a potential option for managing VANETs.

Clustering architecture is one of the most deployed mechanisms that has been used to enhance the reliability, and prolong the lifetime of VANET. In cluster-based VANET, vehicles are divided into small clusters according to some rules, where vehicles in each cluster are managed by the $\mathrm{CH}$ that is in charge of the entire communication operation in the cluster, spectrum management and medium access control. The architecture provides many advantages over other architectures. These include:

- Data aggregation: $\mathrm{CH}$ gathers data from all vehicles in a cluster to eliminate redundant information and generate the final road status and conditions.

- Communication overhead minimization: each node needs to communicate only with $\mathrm{CH}$.

- Easy to manage: each road is divided into segments and a $\mathrm{CH}$ is selected to manage each segment.

- Enhancing network scalability and bandwidth management efficiency. 
In VANET, the selection of a $\mathrm{CH}$ is a pivotal step that significantly influences the reliability and lifetime of the network. In fact, $\mathrm{CH}$ selection becomes more challenging especially when the selection process involves multiple criteria such as spectrum price, $\mathrm{CH}$ speed, and centrality.

To prolong the lifetime of the formed clusters and enhance reachability, despite the high mobility of nodes, the clustering scheme should be completely decentralized and capable of constructing a virtual cluster-based topology, aiming at minimizing the reselection of the $\mathrm{CH}$, and evade rebuilding the whole network.

A novel multi-attributes decision making scheme is introduced in this paper for efficient selection of $\mathrm{CH}$. Fuzzy logic [5], [6] is adopted in this scheme to automate the determination of an appropriate $\mathrm{CH}$ from a set of alternatives and options. Generally, spectrum scarcity is the most serious challenge in VANET [4]. This is due to a dramatic increase in the number of infotainment applications such as multimedia based services, and phone support via an online store, and video streaming [2-4]. To cope with this challenge, many countries allow users to access the unused spectrum provided that they make monetary payment to spectrum owners [4].

Our key contributions in this paper are threefold. First, we propose a new distributed clustering algorithm focused on VANET's lifetime and link stability. Lifetime is mainly defined by the operation time of $\mathrm{CH}$ and cluster, and the low rate of cluster reconstruction. Maintaining link stability is an important metric for measuring the performance of VANET, since nodes tend to be disconnected in a dynamic topology. Link stability indicates the robustness and the longevity of the link between nodes.

Prolonging lifetime of VANET is achieved by adopting the Sugeno fuzzy integral for selecting $\mathrm{CH}$. Our clustering scheme will form clusters with minimum cost, high quality of service (QoS) and relatively minimal velocity between each $\mathrm{CH}$ and its members. Second, to deal with the spectrum scarcity, a spectrum trading scheme is proposed to motivate spectrum owners to hire out their free spectrum. Spectrum availability for VANETs in urban scenarios is crucial for devising an efficient VANET network. Third, QoS provisioning is guaranteed by efficiently utilizing the spectrum resource of a licensed band and selecting a $\mathrm{CH}$ that is able to provide good service for cluster members.

The rest of this paper is organized as follows. Section II presents related work. Section III outlines a VANET model and assumptions for a typical highway scenario. Section IV introduces a new fuzzy control scheme for vehicles clustering. Section V validates the accuracy of the proposed scheme using a simulator and analyzes the capabilities of adopting the fuzzy scheme for prolonging VANET lifetime. Finally, Section VI concludes this paper.

\section{RELATED WORK}

Currently, VANET is the most popular instance of intelligent transportation systems proposed to promote road safety, and also offer smart and entertainment applications as well. Unfortunately, they are faced with more challenges compared to other wireless networks, due to high mobility of vehicles, absence of architectures and the highly dynamic network topology. Furthermore, nodes in VANET must contend with limited spectral bandwidth. In such challenging communication environments, infrastructure based VANETs are able to provide quick solution for most of these problems. The use of access points in VANETs facilitates the management of network resources in a relatively simple manner and enhances connectivity. In order to reduce the costs of deploying a large number of access points in large-scale deployments in the real world and to achieve the benefits of infrastructure-based VANETs, a hierarchical network topology, termed clustering, has been suggested. In this case, nodes are divided into virtual groups using clustering algorithms, and for each group, one node $(\mathrm{CH})$ is elected as a centralized entity to manage intracluster communication arrangements.

\section{A1. Clustering in VANET}

Nowadays, there are many important applications that adopt clustering architecture to manage communication in VANET. Clustering aims to enhance scalability and reliability in the network by converting the network structure from flat into hierarchical one [47]. In Cluster-based VANET, virtual network infrastructure is created without the need for a traditional dedicated expensive wireless network infrastructure. Many security applications [48], [49] have exploited clustering architecture for detecting certain intrusion and threats in VANETs. In order to mitigate scalability in VANET, many routing protocols have exploited clustering architecture [50], [51]. More efficient spectrum management is one benefit facilitated by cluster-based VANETs. Thus, various medium access control (MAC) protocols have adopted clustering architecture since it enables $\mathrm{CH}$ to manage spectrum access among nodes efficiently. CHs were widely used to coordinate spectrum access among nodes [52]. In safety applications, clustering architecture has been proposed to enable fast dissemination of safety messages [4]. Furthermore, $\mathrm{CHs}$ were widely used for quality of service (QoS) assurance [53]. Recently, a great deal of emphasis has been placed exploring clustering in VANET. However, clustering algorithms have yet to overcome some challenges related to the high dynamicity of VANET.

\section{A2. Clustering metrics}

Several cluster algorithms have been proposed to divide vehicles into clusters and select a $\mathrm{CH}$ with the aim of enhancing reliability and network life time. These algorithms have to be robust to nodes mobility where they frequently leave or join clusters. In fact, the key success of VANET revolves around maintaining its stability, where the clustering scheme is a key element to provide reliable communication in VANET. Frequent cluster collapse and sudden changes in cluster topology are among the most challenging issues in clustering algorithms. Authors in [7] proposed a new architecture for VANET based on multi-hop clustering. In the proposed clustering scheme, $\mathrm{CH}$ is selected based on its speed and connectivity with other nodes. In [8], a cellular network is used to transmit vehicles' data. Furthermore, the base station selects a $\mathrm{CH}$ based on the number of hops. A software-defined networking (SDN) enabled 5G VANET is used in [9] to support the increasing traffic and to improve VANET management. VANET is divided into clusters according to road conditions. 
Throughput rate and moving speed are used to select $\mathrm{CH}$ for each cluster. In order to prolong cluster lifetime in VANETs, a genetic algorithm is used in [10] to select a suitable CH. A Dhop clustering algorithm is used in [11] to create clusters with an adaptive size according to the mobility of vehicles. Each vehicle has at most $\mathrm{D}$ hops to connect with the $\mathrm{CH}$. Each vehicle selects its $\mathrm{CH}$ based on relative mobility.

Several metrics are used in [12] to select $\mathrm{CH}$ for urban scenarios. These metrics include vehicle's moving direction, relative position, and link lifetime estimation. In addition, authors proposed a new clustering scheme for VANET in [13]. The main concern of the proposed scheme is maintaining the stability of clusters in VANET. The proposed scheme considers the distance and velocity as criteria for creating a stable clusters structure. Under another umbrella, authors in [14] proposed a clustering scheme for VANET. The proposed scheme utilizes the affinity propagation algorithm for forming clusters and considers typical vehicular mobility in order to maintain the stability of clusters.

Authors in [15] analyzed the performance of VANET with clustering architecture. They presented an analytical model for VANET that incorporates MAC protocol operations, channel conditions, and the moving pattern of vehicles. In [16], authors addressed low efficiency of cluster-based communication. A coalition-based clustering strategy was proposed. The proposed scheme considers the relative velocity, relative position, and the bandwidth availability ratio for cluster formation. The vehicles decide whether to join a cluster or not, based on the coalition utility. In [17], a new clustering technique was proposed for VANET on highways with the aim of enhancing clusters stability. The proposed scheme takes into account speed difference as a parameter to create a relatively stable cluster structure for cluster formation. A new method for estimating traffic volume for VANET was proposed in [18]. Specifically, traffic information is collected from different groups of vehicles using a new clustering algorithm. This information is disseminated to the roadside cloud. $\mathrm{CH}$ is selected based on its position, average speed, and neighborhood degree (i.e. number of neighbors).

In [19], the neighborhood relationship between vehicles is considered for $\mathrm{CH}$ selection. Each vehicle selects the most stable node as its $\mathrm{CH}$. The relative mobility attribute is used to quantify the stability of vehicle. In [20], authors proposed a unified framework for vehicles clustering in VANET. The framework is composed of three important parts, namely, neighbor sampling, $\mathrm{CH}$ selection, and cluster maintenance. Three clustering metrics were used to select $\mathrm{CH}$, namely: vehicle relative position, relative velocity, and link lifetime. VANET and 3G networks were integrated in [21] using mobile gateways (i.e. vehicles). The vehicles are clustered dynamically in VANET according to different metrics including received signal strength, the direction of movement of vehicles and their inter-vehicular distance.

In [22], the sensors attached to vehicles were used to share road status among vehicles and roadside infrastructure. Several metrics were used to group vehicles into clusters. These metrics include: velocity, number of neighbors, and connectivity. Authors proposed a clustering scheme to increase throughput of VANET in [23]. CH manages the spectrum and assigns free channels to cluster members. Three algorithms were proposed in [24] for extending VANETs' lifetime. These algorithms include: Cluster-Based Lifetime Routing (CBLTR) protocol, Intersection Dynamic VANET Routing (IDVR) protocol, and Control Overhead Reduction Algorithm (CORA). In the CBLTR protocol, a $\mathrm{CH}$ is selected based on maximum Lifetime of VANET. The main concern of the IDVR protocol is extending route stability, increasing average throughput of VANET, and reducing delay. The CORA algorithm attempts to reduce control overhead messages that are required for clusters formation. A centralized clustering scheme was proposed in [25]. The scheme integrates IEEE 802.11p and LTE to achieve clustering and to coordinate message delivery. Regional information is collected to perform centralized clustering.

The recent years have witnessed a tremendous rise in VANETs' applications leading to increasing demand for spectrum. Thus, the spectrum availability is one of the most important challenges while deploying a real VANET [54]. For clusterbased VANET, $\mathrm{CH}$ have to pay for getting spectrum. As a result, nodes need to pay the $\mathrm{CH}$ for spectrum access. Thus, the spectrum price becomes one of the most important criteria for $\mathrm{CH}$ selection.

\section{A3. Emerging applications of the proposed clustering scheme}

Some VANET applications require significant computing power and have constrained time delays. However, the computational capabilities of vehicles are limited [62]. Cloudbased VANETs is a promising technology that has been adopted to improve the performance of VANET applications. In [39], authors proposed a cloud-based mobile-edge computing (MEC) off-loading framework in VANET to reduce the latency and transmission cost of computation off-loading. MEC servers predict the processing time to complete the offloading vehicles' tasks. The design and performance of a new distributed and adaptive resource management controller that adopts Cognitive Radio and soft-input/soft-output for data fusion in VANET was performed and conducted in [40].

Energy and computing-limited car smart phones are used by the controller to utilize available Vehicular-to-Infrastructure WiFi connections for performing traffic offloading towards local or remote Clouds.

Recently, researchers have begun investigating artificial intelligence methods at the network edge to improve communication reliability, quality of resource allocation in MEC, make fast and accurate decisions, support QoS provision, optimize the mobile edge caching and collaborative computation offloading, and preserve network security and data privacy [55]-[59],[62].

Our management scheme supports a computation transfer VANET, by utilizing available Vehicular-to-Infrastructure WiFi connections. In order to obtain results faster, a $\mathrm{CH}$ splits up the task and distributes smaller ones between cloud-based mobile edge computing and automobile terminals.

The goal of VANET offloading is to relay cellular networks' data though VANET [61]. The coverage of cellular networks will be extended using the offloading mechanism, and the expense for using the cellular network will be reduced. In [41], authors proposed new cooperative traffic transmission algorithm in a joint $4 \mathrm{G}$ cellular network and VANET network where VANET nodes cooperate with the $4 \mathrm{G}$ cellular network 
infrastructure by routing a portion of the cellular traffic. Furthermore, an analytical study was presented to quantify how much traffic can be offloaded from a $4 \mathrm{G}$ cellular network. In [42], a new model was proposed to evaluate the offloading potential of using VANET from the cellular network. An analytical study was presented in [43] to discuss the capacity of the VANET. Link quality was considered in the proposed model. Our architecture supports relaying cellular network traffic by utilizing unused spectrum for offloading and providing stable VANET architecture. For VANET offloading, a $\mathrm{CH}$ can serve as a local central management entity to perform offloading communication arrangement.

Ultra-dense networks (UDNs) were proposed to meet the explosively increasing demands on spectrum [45]. One of the solutions for addressing the demand for high speed wireless communication services in fifth generation mobile communication networks (5G), is ultra-dense networks (UDNs), which are now deploying small cell stations. Authors in [44], proposed a new scheme for maximizing capacity of UDNs. The main goal of the proposed scheme is finding the best strategy for delivering content either over long range connectivity or short range, while guaranteeing the QoS constraints. Furthermore, authors suggested an algorithm for traffic offloading with orthogonal channels and spectrum allocation. Authors in [45] studied mobile edge computation offloading (MECO) in UDN and proposed a new heuristic greedy offloading scheme as a solution. The results showed the need for superior performance in conducting computation offloading over multiple MEC servers. However, the massive deployment of small base stations will certainly increase co-cell interference, which degrade network performance significantly. Our scheme may be used to simplify the topology of UDNs besides resource management, and interference cancellation in UDNs.

There are significant differences in our approach not only due to considering the spectrum cost for selecting $\mathrm{CH}$ but also due to studying the impact of selecting $\mathrm{CH}$ on the stability of clustering architectures in VANET, under different conditions. The problem of selecting a $\mathrm{CH}$ from a number of competing candidates is a discrete decision making problem in which multiple conflicting criteria have to be considered simultaneously. Our proposed $\mathrm{CH}$ selection scheme presumes a trade-off between different criteria. A trade-off between the multiple objectives of the $\mathrm{CH}$ selection problem is usually inevitable. Hence, to meet one objective, we usually have to give up another objective. For this problem, we are interested in searching for a robust pareto-optimal solution that represents a trade-off between the various objectives of the $\mathrm{CH}$ selection problem. Conflicting criteria (i.e. objectives) are evaluated first. Then, an aggregator operator is used for blending different criteria for optimum selection of CHs. In our work, the fuzzy Sugeno integral is used for the first time, as an aggregation operator to deal with the inconsistent ranking problem of $\mathrm{CHs}$ caused by conflicting criteria.

VANET faces the challenge of spectrum shortage [26]. To tackle this challenge, we combine spectrum trading and the VANET architecture, where the spectrum can be hired out to drivers. This combination resolves the spectrum scarcity problem and supports a wide range of applications such as improving road safety, internet access, multimedia (e.g., audio/video), and data applications. We illustrate the trade-off between the stability of VANET and the cost of spectrum. Previous studies [7-25] assumed spectrum availability for VANET. Moreover, they assumed unlicensed access to spectrum is free and spectrum owners do not get any financial benefits. Therefore, spectrum owners are not motivated to share their spectrum since they are not compensated for spectrum usage. In our work, each $\mathrm{CH}$ has unused spectrum with certain probability, which it would like to lease to drivers. Drivers prefer a $\mathrm{CH}$ that offers the lowest spectrum price, which results in price competition among $\mathrm{CHs}$. However, if a $\mathrm{CH}$ quotes a low price, the likelihood of leasing spectrum increases, but at the cost of generating less revenue.

In our scheme, the game theory is adopted to model the price competition problem. Our model captures uncertain spectrum availability, and performs significant and faster recovery in response to topology changes. This requires efficient clusters configuration. Furthermore, the scheme should consider different attributes of candidates for selecting $\mathrm{CH}$ such as spectrum price, signal strength, stability, velocity, centroid, quality of service, and reachability. In our work, we holistically address all these attributes by devising an adequate adaptive clustering management mechanism.

In summary, an alternative clustering architecture is proposed for prolonging VANETs' lifetime, where stable clusters are formed based on radio environment fluctuations and uncertainty, as well as the heterogeneity of environment parameters such as, QoS requirements, clustering cost, speed, location, direction, etc. Consequently, it is vital to adopt a fuzzy logic based $\mathrm{CH}$ selection algorithm to create hierarchical architecture that considers both architecture cost and QoS requirements. The proposed architecture provides more effective support for diverse applications such as spectrum trading, UDNs, intersection collision avoidance, safety applications, entertainment, and traffic jam warning. The proposed scheme integrates the economical use of the radiofrequency spectrum, QoS constraints, and features of wireless network into a ubiquitous technology.

\section{SYSTEM MODEL}

The roads in the city are modeled as graph G. Each road is assumed to be unidirectional with multiple lanes. The graph $\mathrm{G}$ consists of edge set $\mathrm{E}$ and vertex set R. Edge $e_{i, j}$ represents the road over intersection $r_{i}$ and intersection $r_{j}$. Each road is divided into segments and each segment is divided into cells.

We assume that the arrival of vehicles at the edge $e_{i, j}$ follows a Poisson process with arrival rate $\lambda_{i, j}$. Cell $c_{i}$ is modeled as $\mathrm{ON} / \mathrm{OFF}$ alternating renewal process, ON period indicates that the $i^{\text {th }}$ cell is occupied and OFF period implies that the cell is unoccupied. $s_{i}$ is the state of $i^{\text {th }}$ cell and it is defined as:

$s_{i}=\left\{\begin{array}{l}1, \text { cell } i \text { is occupied (i. e., ON), } \\ 0, \text { cell } i \text { is unoccupied (i. e., OFF), }\end{array}\right.$

Current vehicular density $d_{i}$ for link $e_{i, j}$ is computed as follows: 
$d_{i}=\frac{\sum_{i=1}^{m} s_{i}}{m}$

where $m$ is the number of cells in link $e_{i, j}$. Link status vector is defined as:

$\mathrm{L}(s)=\left\{s=\left(s_{1}, s_{2}, s_{3}, \ldots, s_{m}\right)\right.$,

An example of the system model is shown in Fig. 1. Three clusters, in each, the $\mathrm{CH}$ leases spectrum from roadside mesh points/base station (BST) to serve cluster members. The $\mathrm{CHs}$ act as gateways towards the Radio Network Controller (RNC) and beyond.

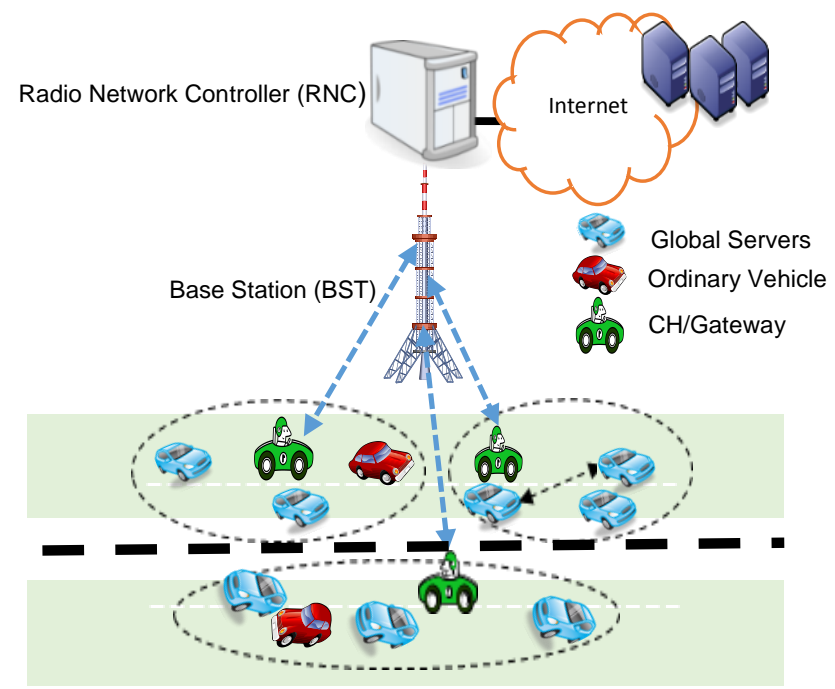

Fig 1. An example of the envisioned architecture where three $\mathrm{CHs}$ lease spectrum from roadside mesh points/ BST) to serve three clusters.

Vehicles move in the city at a random and slowly changing speed $b$, where $b \in\left[b_{\text {min }}, b_{\text {max }}\right]$. Each driver decelerates when the traffic reaches critical vales $\lambda_{i, j}{ }^{C}$ as follows:

$\hat{b}=\max \left[b_{\min }, b_{\max }\left(1-\frac{\lambda_{i, j}}{\lambda_{i, j}{ }^{C}}\right)\right]$

Each vehicle moves straight until it reaches another section where a driver has to choose the next direction to north, south, east and west with probability $Z_{n}, Z_{s}, Z_{e}, Z_{w}$. The following condition must be met:

$Z_{n}+Z_{s}+Z_{e}+Z_{w}=1$

In VANET, each vehicle has a unique ID, which can be the MAC address of the node. VANET infrastructure is represented by a wireless mesh network where each $\mathrm{CH}$ (i.e. mobile gateways) is a mesh router. For clear exposition, the primary notations used in our model are summarized in Table I.

\section{Proposed Cluster-Based RoAd Management}

In this section, we present our proposed cluster management scheme and a detailed algorithm for traffic conditions dissemination system. In our scheme, the traffic management system is conducted on two levels: local (cluster) and global (the whole VANET). Road information is managed as follows:

- Step 1 : Every vehicle gathers real-time traffic information.

- Step 2: All vehicles send their detection results to a $\mathrm{CH}$.

- Step $3: \mathrm{CH}$ combines detection results from all vehicles and generates link status vector.

- Step 4: $\mathrm{CHs}$ exchange link status vectors and then a final decision is made at each $\mathrm{CH}$ using the logical OR operation.

- Step 5: A new status of link is broadcasted to all vehicles in the cluster.

TABLE I

LIST OF RELEVANT NOTATION

\begin{tabular}{|c|c|}
\hline Notations & Description \\
\hline$G(E, R)$ & the underlying road network \\
\hline$e_{i, j}$ & a network edge \\
\hline$r_{i}$ & $i^{\text {th }}$ intersection \\
\hline$\lambda_{i, j}$ & Vehicles arrival rate \\
\hline$c_{i}$ & $i^{\text {th }}$ cell \\
\hline$s_{i}$ & state of $i^{\text {th }}$ cell \\
\hline $\mathrm{L}(s)$ & Link status vector \\
\hline$B$ & vehicle's speed \\
\hline$Y_{i}$ & neighbors set for node $i$ \\
\hline$S(i, j)$ & received signal power at node $i$ from $j^{\text {th }}$ node \\
\hline$d_{i, j}$ & distance between the node $i$ and the neighbor $j$ \\
\hline$\Delta A_{i}$ & rate of $\mathrm{CH}$ 's position. \\
\hline$p_{k}$ & Probability that a $\mathrm{CH}$ has $K$ channels \\
\hline$C_{s}$ & cost of renting spectrum \\
\hline$v_{s}$ & Spectrum valuation \\
\hline$U\left(p_{s}^{i}\right)$ & utility function for $i^{\text {th }} \mathrm{CH}$ \\
\hline$P_{s}^{-i}$ & vector of all prices for $\mathrm{CHs}$ except $i^{\text {th }} \mathrm{CH}$ \\
\hline$F(y)$ & probability of offering price $y$ \\
\hline$K$ & total number of channels \\
\hline$N_{s}$ & total number of $\mathrm{CHs}$ ' channels \\
\hline$P\left(K, N_{s}\right)$ & probability all CHs have spectrum $N_{S}$ \\
\hline$L_{s}$ & The lowest price \\
\hline$p_{n}^{i}$ & evaluation value of spectrum price \\
\hline$d_{i}^{r}$ & packet delivery ratio \\
\hline$Q_{k}$ & service quality for $k^{\text {th }} \mathrm{CH}$ \\
\hline$\check{S}$ & size of spectrum \\
\hline$R_{i}$ & reachability of $i^{\text {th }} \mathrm{CH}$ \\
\hline$O_{i}$ & number of neighbors of the $i^{\text {th }} \mathrm{CH}$ \\
\hline$N_{v}$ & number of vehicles in a cell \\
\hline$\check{C}_{\mathrm{t}}$ & covariance matrix \\
\hline$\varphi \mathrm{r}$ & a state transition matrix \\
\hline $\mathrm{K}_{\mathrm{k}}$ & Kalman gain \\
\hline$D$ & Jacobian of $f\left(\mathrm{x}_{\mathrm{t}}\right)$ \\
\hline$S_{t}$ & signal strength \\
\hline$\Omega$ & set of solutions or alternatives \\
\hline$\psi$ & finite set of criteria \\
\hline$P(C)$ & power set of $C$ \\
\hline$\mu(C)$ & A fuzzy measure on $C$ \\
\hline$\gamma$ & minimum detectable signal power \\
\hline
\end{tabular}


Upon receiving a new link status, drivers may take one of the following actions:

- Accelerate the vehicle speed.

- Decelerate the speed of vehicle.

- Change the route to destination.

\section{A. Proposed Control Clustering Scheme}

Our clustering scheme consists of neighbor discovery, a cluster setup, and cluster formation phase that will be addressed, in turn, as follows.

\section{A.1 Neighbor Discovery:}

VANET is one type of mobile ad hoc network and neighbors are a set of nodes that exist within radio range of an available node. Our clustering scheme is divided into rounds. Each vehicle moving on the road broadcasts a message that contains the node ID at the beginning of each clustering round. Here, we assume each node has a unique ID, which can be the MAC address of the node. Each $\mathrm{CH}$ generates a table that contains neighbors' IDs and the details criteria related to each node. Criteria related to each neighbor node include: received signal strength, speed of vehicle, and vehicle location. The neighbors set, for node $i$ is defined as follows:

$Y_{i}=\{j \mid S(i, j) \geq \gamma\}$

where $S(i, j)$ is the power of the received signal at node $i$ from $j^{\text {th }}$ node. When a node $i$ hears a beacon from neighbor $j$, it gets a triple stimulus : $X_{i, j}=\left[t, d_{i, j}, \mathrm{P}_{j}\right]$, where $t$ is the current time, $d_{i, j}$ is the distance between the node $i$ and the neighbor $j$ and $\mathrm{P}_{j}$ is the position of node $j$. Each vehicle receives a sequence of such triples. Our scheme estimates a new position of candidate $\mathrm{CH}$ using a Kalman filter.

\section{A.2 Cluster setup phase}

VANET is partitioned into multiple segments. Each segment has a $\mathrm{CH}$ responsible for data aggregation and alert message dissemination. The performance of VANET depends on $\mathrm{CH}$ selection. For each segment in the link, the $\mathrm{CH}$ is rotated periodically among existing vehicles in the segment. In order to select a $\mathrm{CH}$, most existing clustering schemes only consider position, speed, and direction of vehicles regardless of spectrum cost, and QoS level. Furthermore, they neglect spectrum management at each cluster. Additionally, most of these schemes mistreat collaborative data processing between nodes. In our work, each node has freedom to become $\mathrm{CH}$. At this phase, each candidate node broadcasts a message to its neighbors if it tends to become a $\mathrm{CH}$. The message contains the input parameters for $\mathrm{CH}$ selection. Our scheme considers the following attributes:

1) Speed of vehicle: Ideal $\mathrm{CH}$ is one with the lowest speed. $\mathrm{A}$ slower vehicle would stay longer within the confines of the cluster compared with a higher speed vehicle. Slow-moving $\mathrm{CH}$ covers less distance and has more stability than other CHs. Each $\mathrm{CH}$ knows its speed. The evaluation value of vehicle's speed is defined as follows: $b_{n}=\frac{b-b_{\min }}{b_{\max }-b_{\min }}$

2) Stability of $\mathrm{CH}$ : Our scheme prioritizes a highly stable $\mathrm{CH}$. A stable $\mathrm{CH}$ has the relative lowest velocity. The velocity of $i^{\text {th }}$ $\mathrm{CH} M_{i}$ is calculated as follows:

$M_{i}=\frac{\Delta A_{i}}{t}$

where $\Delta A_{i}$ is the rate of change in position of the $i^{\text {th }} \mathrm{CH}$. The normalized velocity $M_{n}$ is computed as follows:

$M_{n}=\frac{M-M_{\min }}{M_{\max }-M_{\min }}$

where $M_{\max }$ is the maximum velocity, and $M_{\min }$ is the minimum velocity.

3) Spectrum price: Each $\mathrm{CH}$ informs the drivers about the prices of spectrum. Our scheme prioritizes the $\mathrm{CH}$ that offers the lowest price. In our work, time is divided into slots of equal duration $t$. In every slot $t$, each $\mathrm{CH}$ has $K$ channels with probability $p_{k}$. We assume the probability of spectrum availability is the same for all $\mathrm{CHs}$. Each $i^{\text {th }} \mathrm{CH}$ leases unused spectrum for drivers in return for an access fee of $p_{s}^{i}$. Leasing spectrum incurs a cost $C_{s}$. We assume the truth valuations for spectrum is $v_{s}$. Clearly, no driver will pay more than his valuation (i.e. $p_{s}^{i} \leq v_{s}$ ).

When drivers are interested in hiring spectrum from $\mathrm{CHs}$ that offers a lower price, more drivers are attracted, resulting in price competition among $\mathrm{CHs}$. Although lowering prices makes it difficult for CH's competitors as they are unable to make a profit, it is at the cost of reducing CH's profit. This is a common feature in an oligopoly market, where a buyer sells common goods to a pool of customers. Generally, the Cournot game and the Bertand game are widely used in economics to study and to model competition among sellers for buying goods for clients [35]. In the Bertand game, the clients rent a spectrum from a $\mathrm{CH}$ that quotes the lowest price [36]. Therefore, it is more relevant to our spectrum market.

In our model, the competition among $\mathrm{CHs}$ is formulated using game theory where $\mathrm{CHs}$ are players that interact with each other. The action of $i^{\text {th }} \mathrm{CH}$ is $p_{s}^{i}$. The satisfaction level for each $\mathrm{CH}$ is a numerical value which is measured using a utility function which is the CHs' profit in our work. The utility function for the $i^{\text {th }} \mathrm{CH}$ is defined as follows:

$U\left(p_{s}^{i}\right)=\left\{\begin{array}{c}n *\left(p_{s}^{i}-C_{s}\right), \text { if ith } \text { CH leases } n \text { spectrum units } \\ 0, \quad \text { otherwise }\end{array}\right.$

This game is symmetric since all CHs have the same strategy set (i.e. set of spectrum prices), and the expected utility of adopting a given strategy depends only on the strategies being adopted, regardless of a $\mathrm{CH}$.

Definition 1. For a game with $\mathrm{N} C H s$, the normal-form representation specifies for each $i^{\text {th }} \mathrm{CH}$ a set of strategies $\left\{P_{s}^{i}\right\}$ and an expected utility function $U\left(p_{s}^{i}\right)$. In our work, price 
competition is modeled by a game that is denoted by the tuple $\left[N, \quad\left\{P_{s}^{i}\right\}, U\left(p_{s}^{i}\right)\right]$.

The strategy for the $i^{\text {th }} \mathrm{CH}$ is the selected spectrum price $p_{s}^{i}$ which is selected randomly from a price set using an arbitrary distribution function $P_{s}^{i}($.$) . The vector P_{S}^{-i}$ denotes the vector of all prices for $\mathrm{CHs}$ except $i^{\text {th }} \mathrm{CH}$. Each $\mathrm{CH}$ selects its price randomly from a set of prices using an arbitrary distributed function. Assume $E\left(P_{s}^{i}, P_{s}^{-i}\right)$ denotes the expected utility for the $i^{\text {th }} \mathrm{CH}$ when it selects $P_{s}^{i}$ strategy and other $\mathrm{CHs}$ adopt $P_{s}^{-i}$. Nash equilibrium (NE) is a strategy where no $\mathrm{CH}$ changes its own strategy to gain more profit. The NE for the $i^{\text {th }} \mathrm{CH}$ can be defined as follows:

$E\left(\breve{P}_{S}^{i}, \breve{P}_{s}^{-i}\right) \geq E\left(\hat{P}_{s}^{i}, \hat{P}_{s}^{-i}\right) \quad, \forall \hat{p}_{s}^{i}$

If the demand for spectrum is greater than or equal to the available size of spectrum, then $\mathrm{CH}$ can rent its spectrum even if it chooses the maximum possible price $p_{s}^{i *}$. Thus, each $\mathrm{CH}$ will select $p_{s}^{i *}$ to maximize its profit. $p_{s}^{i *}$ is unique NE for all $\mathrm{CHs}$ when the spectrum demand is greater than supply. Since $\mathrm{CHs}$ are willing to maximize their profits, we assume the offered size spectrum is greater than the required size.

Theorem 1. In this game, there is no deterministic strategy NE where spectrum price is selected dynamically based on the utility function.

Proof. In the Bertrand game, each $i^{\text {th }} \mathrm{CH}$ selects the price $p_{s}^{i}$ deterministically when $p_{k}=1$ which is a unique NE pure strategy. However, this strategy is not NE in our game since the expected utility is 0 if the $\mathrm{CH}$ offers any price greater the cost of renting spectrum. $i^{\text {th }} \mathrm{CH}$ attains utility greater than 0 if its bid is above $p_{s}^{i}$ and less than $v_{s}$ since it leases the unused spectrum at least when it is the only $\mathrm{CH}$ that has free spectrum, which happens with any positive probability (i.e. $p_{k}<1$ ). Hence, no deterministic strategy profile is a NE when $\mathrm{CHs}$ select spectrum prices dynamically. $i^{\text {th }} \mathrm{CH}$ may lease its spectrum if for each $j^{\text {th }}$ $\mathrm{CH}$ :

$p_{s}^{i}<p_{s}^{j}, \forall i \neq j$

The expected price for $i^{\text {th }} \mathrm{CH}, \hat{p}_{s}^{i}$, is the selected spectrum price for the available spectrum. Let $E_{P}$ is the set of declared prices by $\mathrm{CHs}$ and $p_{h}^{i}$ denote the $h^{\text {th }}$ expected smallest price of $i^{\text {th }} \mathrm{CH}$ in $E_{P}$. Let $D_{r}$ indicates the number of drivers who opt for the lowest prices. $j^{\text {th }} \mathrm{CH}$ may lease its spectrum if:

$p_{h}^{i}>p_{m}^{j}, \forall i, j \in E_{P}$

Assume $F\left(p_{m}^{\prime}\right)$ is the probability of offering price $p_{m}^{j}$. The probability that a $\mathrm{CH}$ selects $p_{m}^{j}$ is $1-F\left(\hat{p}_{m}^{j}\right)$. The expected utility for the $j^{\text {th }} \mathrm{CH}$ is calculated as follows:

$$
U\left(p_{s}^{i}\right)=n *\left(p_{m}^{j}-C_{s}\right)\left(1-F\left(p_{m}^{j}\right)\right)
$$

For $j^{\text {th }} \mathrm{CH}$, under $\mathrm{NE}$, the selected spectrum price should maximize the utility for $j^{\text {th }} \mathrm{CH}$ and being the optimal response to other CH's prices.

Definition 2. The price competition game is symmetric since all CHs have identical strategy spaces $S_{p}^{1}=\left\{P_{s}^{1}\right\}=S_{p}^{2}=\left\{P_{s}^{2}\right\}=$ $\left.\cdots=S_{p}^{i}=\left\{P_{s}^{i}\right\}\right)$ and $U\left(p_{s}^{i}\right)=U\left(p_{s}^{j}\right)$. Thus, the utility function can be used for any $\mathrm{CH}$ adopting any strategy regardless of the $\mathrm{CH}$.

The $j^{\text {th }} \mathrm{CH}$ tries to avoid selecting a price from less profitable set of prices since this price will generate the lowest profit. Expected utility $U\left(\hat{p}_{m}^{j}\right)$ is the same for all $p_{m}^{j}$ in NE price set (i.e. profitable price set). $F\left(p_{m}^{j}\right)$ can be specified if $U\left(p_{m}^{j}\right)$ is calculated by considering $F\left(v_{s}\right) . \quad F\left(v_{s}\right)$ is the probability that $p_{s}^{i} \leq v_{s}$ which occurs when $h$ or more $\mathrm{CH}$ possess spectrum size $N_{s}$ which is adequate to serve $D_{r}$ drivers. This probability $P\left(K, N_{s}\right)$ is computed as follows:

$P\left(K, N_{S}\right)=\sum_{i=1}^{K}\left(\begin{array}{c}K \\ i\end{array}\right) p_{N_{S}}^{i}\left(1-p_{N_{S}}\right)^{K-i}$

where $K$ is the number of CHs in the VANET, and $p_{N_{S}}$ is the probability that a $\mathrm{CH}$ has unused spectrum. Hence, $F\left(v_{s}\right)=$ $P\left(K, N_{s}\right)$. Following is the constant that the $j^{\text {th }} \mathrm{CH}$ attains for all prices in NE price set (i.e. profitable set):

$n *\left(v_{s}-C_{s}\right)\left(1-F\left(v_{s}\right)\right)=n *\left(v_{s}-C_{s}\right)\left(1-P\left(K, N_{s}\right)\right)$

Thus, $F\left(p_{m}^{j}\right)$ is computed as follows:

$F\left(\tilde{p}_{m}^{j}\right)=1-\frac{n *\left(v_{S}-C_{S}\right)\left(1-P\left(K, N_{S}\right)\right)}{v_{S}-C_{S}}$

The lowest price $L_{p}$ in NE price is obtained when $F\left(p_{m}^{j}\right)=$ 0 and is calculated as follows:

$L_{p}=\left(v_{s}-C_{s}\right)\left(v_{s}-P\left(K, N_{s}\right)\right)$

Thus,

$F\left(p_{m}^{j}\right)=\left\{\begin{array}{c}0, p_{m}^{j} \leq L_{p} \\ \frac{p_{m}^{j}-L_{s}}{p_{m}^{j}-C_{s}}, L_{p}<p_{m}^{j} \leq v_{s}\end{array}\right.$

For each $\mathrm{CH}$, the lowest price $L_{p}$ is lower boundary for profitable price set $\mathrm{P}$. Spectrum price distribution function $p_{s}^{j}(0)$ leads to the above distribution function $\left(F\left(p_{m}^{j}\right)\right)$ for the $h^{\text {th }}$ expected smallest price of CHs' prices. To maximize its profit, a $\mathrm{CH}$ should offer a price less than or equal $p_{m}^{j}$ given that a $\mathrm{CH}$ has unused spectrum. The probability of offering profitable price $P\left(\hat{p}_{h}^{j}\right)$ is computed as follows:

$P\left(\dot{p}_{h}^{j}\right)=p_{N_{s}} P_{s}^{j}\left(\dot{p}_{h}^{j}\right)$ 
The probability that $h$ or more expected prices are offered and they are less than or equal to $\bar{p}_{h}^{j}$ is computed as follows:

$F\left(\begin{array}{c}p_{h}^{j} \\ h\end{array}\right)=\sum_{i=1}^{K-1}\left(\begin{array}{c}K \\ i\end{array}\right)\left[p_{N_{S}} P_{s}^{j}\left(\dot{p}_{h}^{j}\right)\right]^{i}\left[1-p_{N_{S}} P_{S}^{j}\left(\dot{p}_{h}^{j}\right)\right]^{K-i}$

$P_{s}^{j}\left(\dot{p}_{h}^{J}\right)$ can computed from $(21)$ as follows:

$\left(\dot{p}_{h}^{j}\right)=\left(\frac{1}{p_{N_{S}}}\right)^{\prime} \mathrm{P}\left({\left.\stackrel{p}{p_{h}^{j}}\right)}^{\prime}\right)$

$\mathrm{P}\left(\hat{p}_{h}^{j}\right)$ can be calculated by solving the following equation:

$\sum_{i=1}^{K-1}\left(\begin{array}{c}K \\ i\end{array}\right)\left[p_{N_{S}} \mathrm{P}\left(\hat{p}_{h}^{j}\right)\right]^{i}\left[1-p_{N_{S}} \mathrm{P}\left(\hat{p}_{h}^{j}\right)\right]^{K-i}=F\left(\dot{p}_{h}^{j}\right)$

The NE prices are selected according to the following function:

$$
P_{s}^{j}\left(\dot{p}_{m}^{\prime}\right)=\left\{\begin{array}{c}
0, p_{m}^{\prime j} \leq L_{p} \\
\left(\frac{1}{p_{N_{s}}}\right) \cdot \mathrm{P}\left(\hat{p}_{h}^{j}\right), L_{p}<\dot{p}_{m}^{j} \leq v_{s} \\
1, \hat{p}_{m}^{j}>v_{s}
\end{array}\right.
$$

There are many studies of NE existence analysis for the pricing competition problem [37], [38]. The work in [37] expanded the 2-players game to n-players to find NE. NE satisfies the conditions that $\mathrm{P}$ is a compact convex set in an Euclidean space, $P_{s}^{j}\left(p_{m}^{j}\right)$ is a continuous function on $\mathrm{P}$, and $P_{s}^{j}\left(p_{m}^{j}\right)$ is a convex function on $\mathrm{P}$ with respect to $p_{m}^{j}$.

Theorem 2. Given the non-price spectrum $k \in K$ and $p_{m}^{j} \in \mathrm{P}$, non-cooperative game strategies for $N$ CHs' have $\operatorname{NE~} \mathrm{P}\left({ }^{\prime} p_{h}^{j}\right)$.

Proof. First, for each $j^{\text {th }} \mathrm{CH}, \mathrm{P}$ is closed interval. Hence, $\mathrm{P}$, for any $p_{x 1}^{j}, p_{x 2}^{j} \in \mathrm{P}$, there is $\omega p_{x 1}^{\prime j}+(1-\omega) p_{x 2}^{j} \in \mathrm{P}$, for any $\omega \in[0,1]$ and $\mathrm{P}$ is considered as convex set. $\mathrm{P}\left(p_{m}^{j}\right)$ is continuous on $\mathrm{P}$ when $L_{p}<p_{m}^{j} \leq v_{s}$. Suppose $j^{\text {th }} \mathrm{CH}$ adopts the strategy ' $\mathrm{P}\left(p_{m}^{j}\right)$. Recall $\bar{p}_{h}^{j}$ is the smallest $h^{\text {th }}$ price in $E_{P}$. The utility of the $j^{\text {th }} \mathrm{CH}$ is $p_{m}^{j}-C_{s}$ if $L_{p}<p_{m}^{j} \leq v_{s}$, and 0 if $p_{m}^{j}<p_{h}^{j}$ and $\mathrm{F}\left(p_{m}^{j}\right)=P\left(p_{h}^{j} \leq p_{m}^{j}\right)$. The expected utility for each price $p_{m}^{j} \in\left[L_{p}, v_{s}\right]$ is calculated as follows:

$$
\begin{aligned}
E\left(u_{j}\left(\mathrm{P}\left(\dot{p}_{m}^{j}\right), P_{s}^{-j}(.)\right)\right. & =\left(\dot{p_{m}^{j}}-C_{s}\right) P\left(\dot{p}_{h}^{j}>p_{m}^{\dot{j}}\right) \\
& =\left(\dot{p}_{m}^{j}-C_{s}\right)\left(1-\mathrm{F}\left(\dot{p}_{m}^{j}\right)\right) \\
& =\left(L_{p}-C_{s}\right)
\end{aligned}
$$

$j^{\text {th }} \mathrm{CH}$ 's utility for price $p_{x}^{j}$ that is less than $p_{m}^{j}$ is $\left(p_{x}^{j}-C_{s}\right)$ which is less than $\left(L_{p}-C_{s}\right)$. Furthermore, if $j^{\text {th }} \mathrm{CH}$ selects a price $p_{y}^{j} \geq p_{m}^{j}$, then the utility is 0 . Hence, any spectrum price falls in the $\left[L_{p}, v_{s}\right]$ is the best price. Since, $\mathrm{P}\left(\hat{p}_{m}^{j}\right)$ set in $\left[L_{p}, v_{s}\right]$, is the best strategy for a $\mathrm{CH}$ in an NE.

The evaluation value of spectrum price $p_{n}^{i}$ for $i^{\text {th }} \mathrm{CH}$ is defined as follows: $p_{n}^{i}=\left(p_{s}^{i}-p_{\min }\right) /\left(p_{\max }-p_{\min }\right)$

where $p_{\min }$ is the minimum spectrum price, and $p_{\max }$ is the maximum predicted price.

3) Service quality analysis: The likelihood of selecting $\mathrm{CH}$ increases as the signal quality increases. In our scheme, spectrum quality analysis is used to assess the ability of $\mathrm{CH}$ to deliver excellent service in terms of communication. Delivery ratio is used as in [27] to evaluate the $i^{t h}$ channel service quality as follows:

$d_{i}^{r}=\frac{s_{n}}{t}$

where $S_{n}$ is the count of received packets during the last time window $t$. The evaluation value of service quality for $i^{\text {th }} \mathrm{CH}$ is calculated through:

$Q_{i}=\frac{\sum_{j=1}^{H} d_{j}^{r}}{\check{S}}$

where $\check{S}$ is the size of spectrum (i.e. number of channels) at $i^{\text {th }}$ $\mathrm{CH}$.

4) Reachability: Reachability refers to number of input and output links for a $\mathrm{CH}$. It can be interpreted as sign of CHs' ability to serve the maximum number of nodes in VANET. The reachability of $i^{\text {th }} \mathrm{CH} R_{i}$ is measured as follows:

$R_{i}=\frac{O_{i}}{N_{v}}$

where $O_{i}$ is the number of neighbors of the $i^{\text {th }} \mathrm{CH}$ in a cell, and $N_{v}$ is the total number of nodes in a cell. The likelihood of selecting $i^{\text {th }} \mathrm{CH}$ as the cluster head increases as its reachability $R_{i}$ gets closer to 1 .

5) Centroid: Kalman filtering is used to predicate unknown variables using data about the past behavior of the system (i.e. previous state). In our work, a Kalman filter estimates vehicle position every time a new sample of distances is acquired. A $\mathrm{K}$-means clustering algorithm [28] is used to determine the closest $\mathrm{CH}$ to centroid. System state $\mathrm{x}_{\mathrm{t}}$ at time $\mathrm{t}$ is defined as:

$$
x_{t}=\left\{\begin{array}{l}
E_{t}=\left(e_{1}, e_{2}, e_{3}, \ldots, e_{k}\right) \\
W_{t}=\left(w_{1}, w_{2}, w_{3}, \ldots, w_{k}\right),
\end{array}\right.
$$

where $\mathrm{E}$ represents the position vector, and $\mathrm{W}$ is the velocity vector. In our work, we assume each vehicle moves at constant velocity between time-steps, the new state $\mathrm{x}_{\mathrm{t}+1}$ after time $\Delta t$ is computed as follows:

$\mathrm{x}_{\mathrm{t}+1=} \mathrm{E}_{\mathrm{t}}+\mathrm{W}_{\mathrm{t}} \Delta t$

Assume the covariance matrix $\check{C}_{t}$ is $[\mathrm{kXk}$ ] for the Kalman filter. The predicted covariance matrix is calculated as follows:

$\check{\mathrm{C}}_{\mathrm{t}+1}=\varphi \check{\mathrm{C}}_{\mathrm{t}}+\mathrm{O}_{\mathrm{t}}^{\prime}$ 
where $\varphi$ is a state transition matrix, and $\sigma_{\mathrm{t}}^{\prime}$ represents the expectation of degradation in the state vector quality. After computing the predicted state vector and covariance matrix, the newly measured distance sample is processed to improve the accuracy of the measurements.

We assume the estimated variance of distances $R_{k}$ follows the normal distribution. The measurements and predicted state vector are weighted using covariance matrices. Kalman gain $\mathrm{K}_{\mathrm{k}}$ is used for assigning weight to the measurements, if the measurements are inaccurate (large variances) when compared to the estimated state vector. Assume the function $f\left(x_{t}\right)$ computes the predicated distances to the centroid. Let $D$ be the Jacobian of $\mathrm{f}\left(\mathrm{x}_{\mathrm{t}}\right)$ and $\mathrm{M}_{t+1}$ be the measurement at time $t+1$. Given the "old" a posteriori covariance matrix, $\check{C}_{\mathrm{t}}$, state vector $\mathrm{x}_{\mathrm{t}}$, we can compute the "updated" parameters as follows:

$\mathrm{K}_{\mathrm{k}}=D \check{\mathrm{C}}_{\mathrm{t}}^{T}\left(\check{\mathrm{C}}_{\mathrm{t}} D \check{\mathrm{C}}_{\mathrm{t}}^{T}+\mathrm{R}_{\mathrm{k}}\right)^{-1}$

$x_{t+1}=x_{t}+K_{k}\left[M_{t-} f\left(x_{t}\right)\right]$

$\check{\mathrm{C}}_{\mathrm{t}+1}=\check{\mathrm{C}}_{\mathrm{t}}-\mathrm{K}_{\mathrm{k}} D \check{\mathrm{C}}_{\mathrm{t}}$

The evaluation value of predicted distances to the centroid is defined as follows:

$f\left(x_{t}\right)_{n}=\frac{f\left(x_{t}\right)-f\left(x_{t}\right)_{\min }}{f\left(x_{\mathrm{t}}\right)_{\max }-f\left(x_{\mathrm{t}}\right)_{\min }}$

where $f\left(x_{t}\right)_{\text {min }}$ is the minimum predicted distance, and $f\left(x_{t}\right)_{\text {max }}$ is the maximum predicted distance.

(7) Signal strength: Signal strength refers to the signal power at the receiver. The same signal power model that was used in [29] is considered to assess the ability of a $\mathrm{CH}$ to provide good QoS for cluster members in term of transmitting data over a wireless medium. The evaluation value of signal strength is calculated as follows:

$S_{t}=\frac{S_{a v g}}{S_{c}}$

Here $S_{\text {avg }}$ is the average signal strength at neighbors of a $\mathrm{CH}$, and $S_{c}$ is the CH's signal strength at the cluster edge.

\section{A.3 Fuzzy Integral for Candidate CH Evaluation and Cluster formation}

The cluster formation phase aims to create a cluster for each road in a distributive manner. Nodes make independent decisions for selecting $\mathrm{CH}$ using fuzzy integral logic at this phase. Assume the set $\Omega=\left\{\mathrm{CH}_{1}, \mathrm{CH}_{2}, \mathrm{CH}_{3}, \ldots, \mathrm{CH}_{n}\right\}$ represents the set of solutions or alternatives among which the decision maker must choose (i.e. candidate $\mathrm{CHs}$ ). Let $\psi=$ $\left\{C_{1}, C_{2}, C_{3}, \ldots, C_{m}\right\}$ be the finite set of criteria that should be considered for selecting an optimal $\mathrm{CH}$. Each $i^{\text {th }} \mathrm{CH}$ is associated with a vector $V^{i} \in \Omega$ whose components $v_{j} \in$ $\psi, v_{j}$ represent the value of the $j^{\text {th }}$ attribute to be taken into account in the decision making process. Each $j^{\text {th }}$ criterion would correspond to an objective function. Given these criteria, modeling the selection process preferences $\geqslant$ is realized through an overall utility function:

$O: \Omega \rightarrow I R$

Such that:

$\forall a, b \in \Omega, a \geqslant b \Leftrightarrow O(a) \geq O(b)$

The utility functions, denoted $\mathrm{O}_{1}\left(\mathrm{CH}_{i}\right), \ldots, \mathrm{O}_{m}\left(\mathrm{CH}_{i}\right)$ map each criterion of $i^{\text {th }} \mathrm{CH}$ to a single satisfaction scale $\varepsilon \in I R$. The criteria for each $\mathrm{CH}$ need to be aggregated to a single score or rank for $i^{\text {th }} \mathrm{CH}$ as follows:

$\forall C H_{i} \in \Omega, O\left(C H_{i}\right)={ }^{\prime} \mathrm{H}\left(\mathrm{O}_{1}\left(\mathrm{CH}_{i}, \ldots \mathrm{O}_{m}\left(\mathrm{CH}_{i}\right)\right)\right.$

Theorem 3. $\mathrm{CH}$ selection is monotone non-decreasing, if the utility function ' $\mathrm{H}\left(\mathrm{O}_{1}\left(\mathrm{CH}_{i}\right), \ldots \mathrm{O}_{m}\left(\mathrm{CH}_{i}\right)\right)$ is non-decreasing.

Proof. The main concern of the $\mathrm{CH}$ selection scheme is selecting the $\mathrm{CH}$ with the maximum rank as follows:

$C H=\arg \max _{C H_{i} \in \Omega}{ }^{\prime} \mathrm{H}\left(V^{i}\right)$

Suppose the $j^{\text {th }}$ attribute for $i^{\text {th }} \mathrm{CH}$ increases by $\Delta$ where $\Delta>0$. Because of the assumption of monotone hazard rate, clearly the score for $O_{j}\left(C_{j}+\Delta\right)$ is greater than $O_{j}\left(C_{j}\right)$. Therefore, ${ }^{\prime} \mathrm{H}\left(O_{1}\left(C_{1}\right)\right.$, $\left.\ldots, C_{j}, \ldots, O_{m}\left(C_{m}\right)\right) \geq{ }^{\prime} \mathrm{H}\left(O_{1}\left(C_{1}\right), \ldots, C_{j}+\Delta, \ldots, O_{m}\left(C_{m}\right)\right)$ and the selection scheme selects $\mathrm{CH}$ that has the maximum rank ${ }^{\prime} \mathrm{H}(V)$. We use a Fuzzy measure to assign weight for each criterion and for any coalition of criteria.

Definition 3. A solution $\mathrm{CH}_{i}$ is said to be optimal if there does not exist $\mathrm{CH}_{j} \in \Omega$ such that ${ }^{\prime} \mathrm{H}\left(V^{j}\right) \leq{ }^{\prime} \mathrm{H}\left(V^{i}\right)$.

Definition 4. Assume $P(C)$ is the power set of $C$. A fuzzy measure $\mu$ on $C$ is a function $: P(C) \rightarrow[0,1]$, satisfying the following axioms [30, 31]:

- $\mu(\varnothing)=0, \mu(C)=1$

- $X \subset Y \subset C$ implies $\mu(X) \leq \mu(Y)$. (monotonicity)

Sugeno suggested a new $\lambda$-fuzzy measure [30] which satisfies the following properties: $\forall X, Y \in P(C), \mathrm{X} \cap \mathrm{Y}=\emptyset$

${ }^{\prime} \mathrm{H}_{\lambda}(X \cup Y)={ }^{\prime} \mathrm{H}_{\lambda}(X)+{ }^{\prime} \mathrm{H}_{\lambda}(Y)+\lambda^{\prime} \mathrm{H}_{\lambda}(X)^{\prime} \mathrm{H}_{\lambda}(Y), \lambda \in(0,1)$

The mapping function similar to that in [30, 31] for a finite set $\left\{c_{1}, c_{2}, c_{3}, \ldots, c_{m}\right\}$ can be written as follows

${ }^{\prime} \mathrm{H}_{\lambda}\left(\left\{c_{1}, c_{2}, c_{3}, \ldots, c_{m}\right\}\right)=\sum_{i=1}^{m}{ }^{\prime} \mathrm{H}_{i}+\lambda \sum_{i=1}^{m-1} \sum_{j=i+1}^{m}{ }^{\prime} \mathrm{H}_{i}{ }^{\prime} \mathrm{H}_{j}+\cdots+$
$\lambda^{m-1}{ }^{\prime} \mathrm{H}_{1}{ }^{\prime} \mathrm{H}_{2} \ldots .+{ }^{\prime} \mathrm{H}_{n}=\frac{1}{\lambda}\left|\prod_{i=1}^{m}\left(1+\lambda \mathrm{H}_{i}\right)-1\right|$

where ' $\mathrm{H}_{i}$ is fuzzy density and can be written as ${ }^{\prime} \mathrm{H}_{i}={ }^{\prime} \mathrm{H}_{\lambda}\left(\left\{c_{i}\right\}\right)$.

Theorem 4. The mapping function ${ }^{\prime} \mathrm{H}_{\lambda}\left(\left\{c_{1}, c_{2}, c_{3}, \ldots, c_{m}\right\}\right)$ for a finite set of criteria $\left\{c_{1}, c_{2}, c_{3}, \ldots, c_{m}\right\}$ is nonempty, compact, and convex with $\mathrm{O}_{1}\left(\mathrm{CH}_{i}\right), \ldots, \mathrm{O}_{m}\left(\mathrm{CH}_{i}\right)$, continuous in $\left\{c_{1}, c_{2}, c_{3}, \ldots, c_{m}\right\}$ and quasi-concave in $c_{i}$, the optimal choice for a $\mathrm{CH}$ : 
$\mathrm{S}(\mathrm{CH})=\arg \max _{\mathrm{CH}_{\mathrm{i}} \in \Omega}{ }^{\prime} \mathrm{H}\left(\mathrm{V}^{\mathrm{i}}\right)$

is nonempty, convex-valued, and upper hemicontinuous.

Proof. Generally, every compact space is a continuous image of the compact set. Since ' $\mathrm{H}\left(V^{i}\right)$ is the continuous image of the compact set $\left\{c_{1}, c_{2}, c_{3}, \ldots, c_{m}\right\}$, it is compact and has a maximum. So $S(\mathrm{CH})$ is nonempty. $S(\mathrm{CH})$ is convex because the set of maxima of a quasiconcave function ${ }^{\prime} \mathrm{H}\left(V^{i}\right)$ on a convex hull of set $\left\{c_{1}, c_{2}, c_{3}, \ldots, c_{m}\right\}$ which is convex also. To show that $S(\mathrm{CH})$ is upper hemicontinuous, we show that for any sequence $S\left(C^{m}\right) \rightarrow C H_{i}$ such that $C^{m} \in \Psi$ for all $m$, we have $C H_{i} \in \Omega$. To see this, note that for all $m,{ }^{\prime} \mathrm{H}\left(V^{i}\right) \geq$ ${ }^{\prime} \mathrm{H}\left(V^{j}\right), \forall i, j \in \Omega$. So because of continuity of ${ }^{\prime} \mathrm{H}\left(V^{i}\right)$, we have ${ }^{\prime} \mathrm{H}\left(V^{i}\right) \geq{ }^{\prime} \mathrm{H}\left(V^{j}\right)$. Let $\dot{A}$ be the function that represents the value of a particular criteria for the alternatives, while $Y$ is the weight for the criteria.

Sugeno fuzzy integral is used to compute the overall evaluation of $\mathrm{CH}$, similar to [30], [31], [32] Sugeno fuzzy integral can be expressed as follows:

$F(\dot{\mathrm{A}})=\int \dot{\mathrm{A}} d y=\max _{i \in m} \min \left(\dot{\mathrm{A}}\left(c_{i}\right),{ }^{\prime} \mathrm{H}_{\lambda}\left({ }^{\prime} \mathrm{H}_{i}\right)\right)$

Definition 5. A vector $C_{i} \in \Omega$ is a fuzzy-optimal solution of the $\mathrm{CH}$ selection problem if it is a unique optimal solution.

Theorem 5: The CH set generated by the clustering scheme can cover all of the vehicles, and there is at most one $\mathrm{CH}$ within each segment.

Proof. The solution for the $\mathrm{CH}$ selection problem is optimal selection. A solution $\mathrm{CH}_{i} \in \Omega$ is said to be Pareto-optimal if there exists no alternative solution $\mathrm{CH}_{j} \in \Omega$ that improves some of the objective functions for some criteria without degrading at least another objective function. Thus, $\mathrm{CH}_{i} \in \Omega$ is said to be Pareto-optimal solution of $\mathrm{CH}$ selection problem if there exists no other feasible $\mathrm{CH}_{j} \in \Omega$ such that $\mathrm{O}_{k}\left(\mathrm{CH}_{j}\right) \leq \mathrm{O}_{k}\left(\mathrm{CH}_{i}\right), \quad \forall k=$ $1, \ldots, m$ with strict inequality for at least one criteria $k$. Hence, for each segment the $\mathrm{CH}$ selection algorithm allocates only one $\mathrm{CH}$ which can serve all cluster members. The stability of the cluster is defined as the amount of time that the $\mathrm{CH}$ can serve all cluster members (i.e. the amount of time that the $\mathrm{CH}$ can cover its cell in the road). Reachability is defined as the percentage of nodes that received the message out of the total nodes in the cluster. The reachability is monitored and if a $\mathrm{CH}$ fails to cover any location in its cell, another $\mathrm{CH}$ should be elected and the cluster setup phase should be launched.

The coverage probability $\mathrm{p}(\mathrm{S}<\gamma)$ is the probability that a $\mathrm{CH}$ cannot serve a node at distance $d$ and it is computed as follows:

$p(S<\gamma)=\int_{-\infty}^{\gamma} \frac{1}{\sigma \sqrt{2 \pi}} e^{\frac{(S-\mu)^{2}}{2 \sigma^{2}}} d S$

where $\gamma$ is the minimum detectable signal power, $S$ is the signal power at distance $d$. We assume the received power $S$ has a Gaussian distribution with mean $\mu$ and standard deviation.

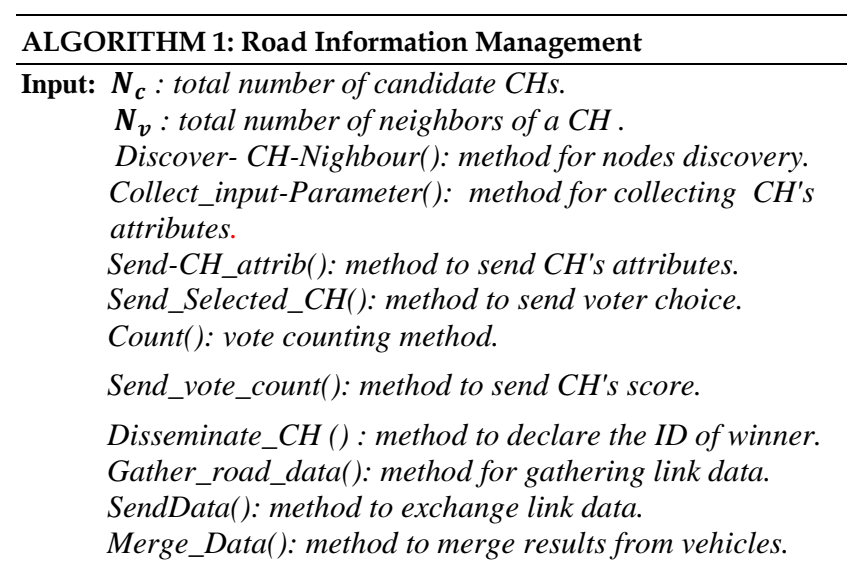

Output: $\boldsymbol{F}_{\boldsymbol{S}}$ : Final link status.

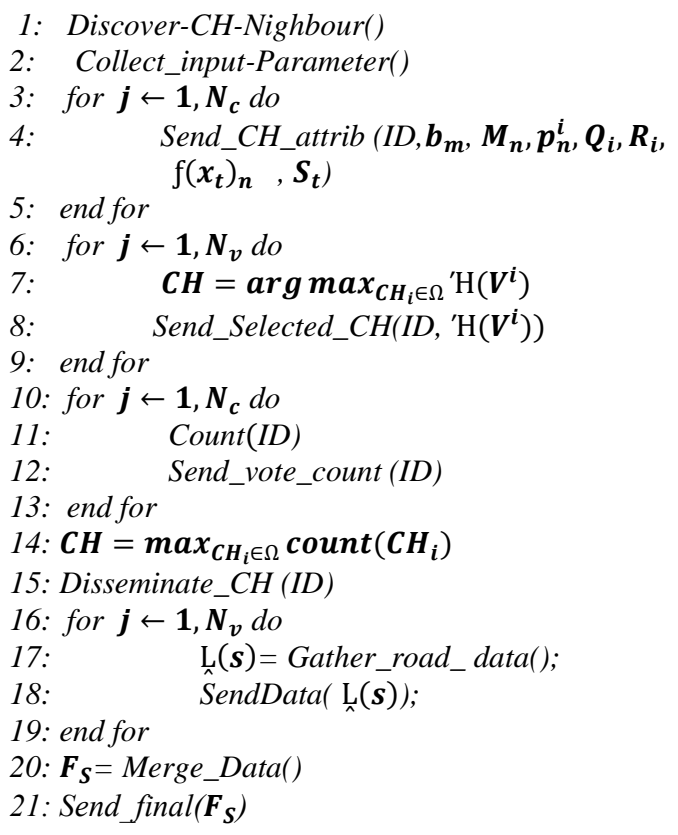

Road information management algorithm is performed in two phases: cluster formation phase, and data broadcast phase. At the beginning, each vehicle broadcasts a message including its speed and location information. Once a node receives messages from all neighboring nodes, it generates a table of neighbors. After that, each node elects itself as a $\mathrm{CH}$ by broadcasting a message to its neighbors. The message contains the overall evaluation of $\mathrm{CH}$ criteria. Each node applies a fuzzy integral approach to the candidates' selection problem. For each road segment, the node with highest overall evaluation becomes the $\mathrm{CH}$. After the creation of the clusters, each road segment is managed by the $\mathrm{CH}$, which is responsible for getting the traffic data from its cluster's nodes and then aggregate them, before sending the aggregates to other $\mathrm{CHs}$ and cluster members. We apply Algorithm 1 for cluster construction and road management. 


\section{PERformance EVAluation}

In this section, the performance of the proposed scheme is evaluated. We consider an urban scenario of $10 \mathrm{~km} \times 10 \mathrm{~km}$, where vehicles are distributed randomly over the road, having speeds of $30-60 \mathrm{~km} / \mathrm{h}$. We simulated a 2-lane per direction highway. Vehicles are equipped with a Global Positioning System (GPS) receiver. Thus, each vehicle knows its own position and velocity. The two-ray ground model is adopted as the radio propagation model.

Each road is divided into equal cells. For each level of traffic density, the vehicles' speed changes uniformly between 30 $\mathrm{km} / \mathrm{h}$ (i.e. low speed) and $60 \mathrm{~km} / \mathrm{h}$ (i.e. high speed). The transmission range is 300 meters. The data rate is set to $6 \mathrm{Mbps}$, the size of the message including the mobility information is 100 bytes. Assume vehicle arrivals are Poisson distributed with an hourly traffic flow of $1800 \mathrm{veh} / \mathrm{h}$. The simulation runs for 800 seconds.

TABLE II

PARAMETERS USED

\begin{tabular}{|c|c|c|}
\hline \multicolumn{2}{|l|}{ Parameter } & Value \\
\hline \multicolumn{2}{|c|}{$\begin{array}{l}\text { Number of messages per } \\
\text { vehicle }\end{array}$} & Random \\
\hline \multicolumn{2}{|c|}{ Type of interface per node } & $802.11 \mathrm{~b}$ \\
\hline \multicolumn{2}{|c|}{ MAC layer } & IEEE $802.11 \mathrm{~b}$ \\
\hline \multicolumn{2}{|c|}{ Transmission power } & 0.1 watt \\
\hline \multicolumn{2}{|c|}{ Packet size } & 512 \\
\hline \multicolumn{2}{|c|}{ Max vehicle speed } & $60 \mathrm{~km} / \mathrm{h}$ \\
\hline \multicolumn{2}{|c|}{ Message cycle } & $100 \mathrm{~ms}$ \\
\hline \multicolumn{2}{|c|}{ Max transmission range } & $300 \mathrm{~m}$ \\
\hline \multicolumn{2}{|c|}{ Area range } & $10 \mathrm{~km} \times 10 \mathrm{~km}$ \\
\hline \multicolumn{2}{|c|}{ Radio Propagation Model } & Tow-ray ground \\
\hline \multicolumn{2}{|c|}{ Data rate } & $6 \mathrm{Mbps}$ \\
\hline \multirow{4}{*}{$\begin{array}{l}\text { Simulatio } \\
\mathrm{n} \\
\text { Device }\end{array}$} & Intel i5 Core & $2.50 \mathrm{GHz}$ \\
\hline & Process cores & $2 \times 2.50 \mathrm{GHz}$ \\
\hline & RAM & $6 \mathrm{~GB}$ \\
\hline & OS & Windows 764 bit \\
\hline
\end{tabular}

We assume the probability of selecting any direction is equal at each intersection, for the driver. The parameters chosen for evaluating the algorithm are shown in Table II. The results are averaged over sufficient independent runs so that the confidence level is $95 \%$ and relative errors do not exceed $5 \%$.

We examine the performance of the proposed scheme under different parameter settings. For VANET, the key performance measures of interest in the simulations are:

(1) Cluster stability: the amount of time that the $\mathrm{CH}$ can serve all cluster members.

(2) Packet loss probability: the probability of unsuccessful transmission of a packet.

(3) Average packet delay: an average time elapsed until a packet is delivered successfully to the destination.

Fig. 2 shows cluster stability as a function of vehicle speed and level of density. As expected, the stability of cluster is maintained under lower values of vehicles' speeds. This is because when a $\mathrm{CH}$ speed is low, it can stay at cell for long time. Hence, the resulting architecture of VANET is stable and exhibits long average duration, low average cluster change, and long average rate of $\mathrm{CH}$ duration.

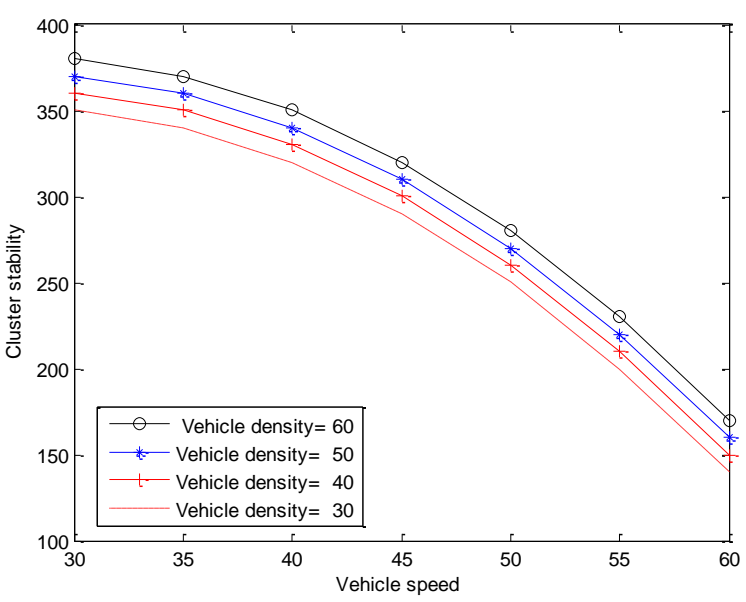

Fig 2. Cluster stability under different vehicle speed

Our scheme prefers $\mathrm{CH}$ with the lowest velocity. Fig. 3 shows the evaluation of CH's ability to serve all cluster members as a function of vehicle speed. The mobility of vehicle (speed) clearly appears to have a strong impact on performance of a $\mathrm{CH}$. Thus, this result should be carefully analyzed: real VANET architectures are affected significantly by the speed of vehicles since it determines $\mathrm{CH}$ duration. The coverage probability decreases as the speed of vehicles increases. When $\mathrm{CH}$ speed is high, it moves away faster from its cluster. In order to maximize $\mathrm{CH}$ duration, our scheme prefers $\mathrm{CHs}$ with lower speed, higher degree of centrality, and lower velocity.

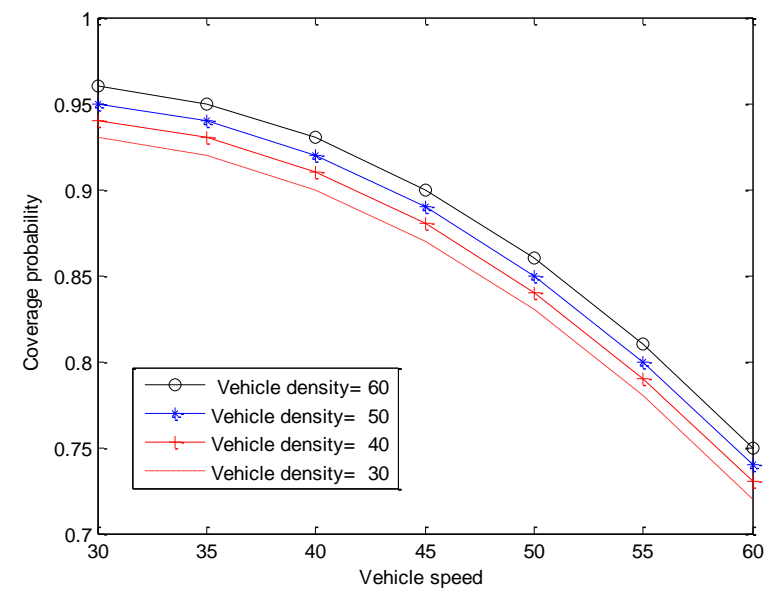

Fig 3. Coverage probablity under different vehicle speed

In our work, the link stability metric indicates the lifetime of the link (i.e. Link connectivity duration) between a vehicle and a $\mathrm{CH}$. It is computed using the proposed model in [46]. From Fig. 4 , we note that if the vehicle density is high then the communication link is stable. Vehicles decrease their speed in congested areas, which increases the link lifetime. However, if vehicle density is low, the link stability between $\mathrm{CH}$ and its clients' decreases. Link breakage rate increases as the speed of the vehicle increases. 


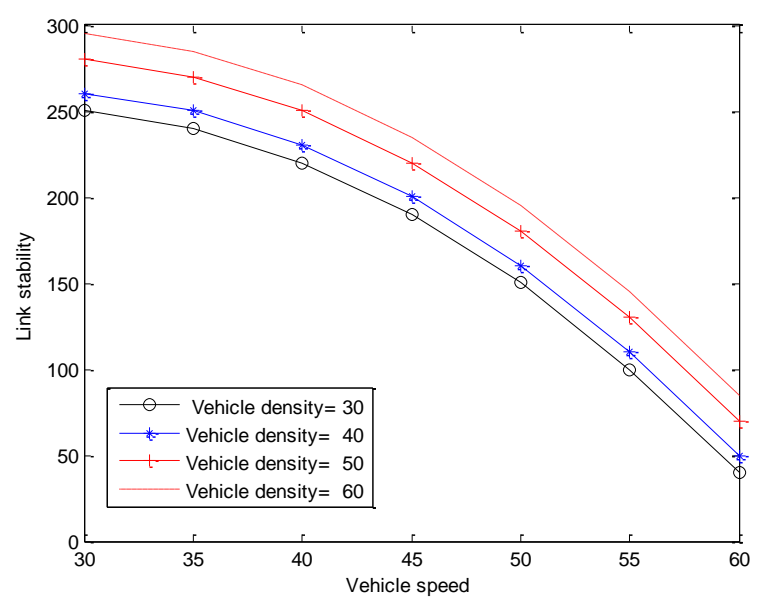

Fig 4. Link stablity under different values of vehicle speed

To study the effect of cluster stability on the performance of VANET, we measure packet loss probability under various values of cluster stability and vehicle density. From Fig.5, we notice that as the lifetime of cluster increases, the packet loss probability decreases. From this experiment, we find that using VANET becomes less efficient when $\mathrm{CH}$ lifetime is short. Clustering architecture has to be reconfigured if $\mathrm{CH}$ leaves a cluster because of high speed and this will significantly increase the message overhead. The dynamic topology of VANET causes cluster collapse and new clusters will be formed. Therefore, traffic will be increased and more bandwidth will be consumed. As a result, packet loss probability will increase significantly. Our scheme mitigates communication overhead by selecting a $\mathrm{CH}$ that stays longer at cell and this creates more stable clusters.

Fig. 6 shows the effect of varying vehicles' speeds and densities on the average packet delay. It can be seen that the delay increases as the speed and density increase. This is because an increment in the number of vehicles increases the volume of data over VANET. Furthermore, the delay increases with the speed of vehicles due to extra clustering overhead. This is expected because increasing the speed of vehicles increases the number of created clusters and this decreases cluster lifetime. Therefore, a $\mathrm{CH}$ will join more clusters during its lifetime, generating more clustering related messages that consume more bandwidth and create additional traffic on VANET. The generated clustering overhead naturally degrades the performance of VANET significantly in terms of packet delay. Spectrum price is one of the most important criteria in determining the $\mathrm{CH}$. High spectrum price may degrade the performance of VANET and increase packet loss rates if the scheme neglects other criteria and gives more weight to price criterion.

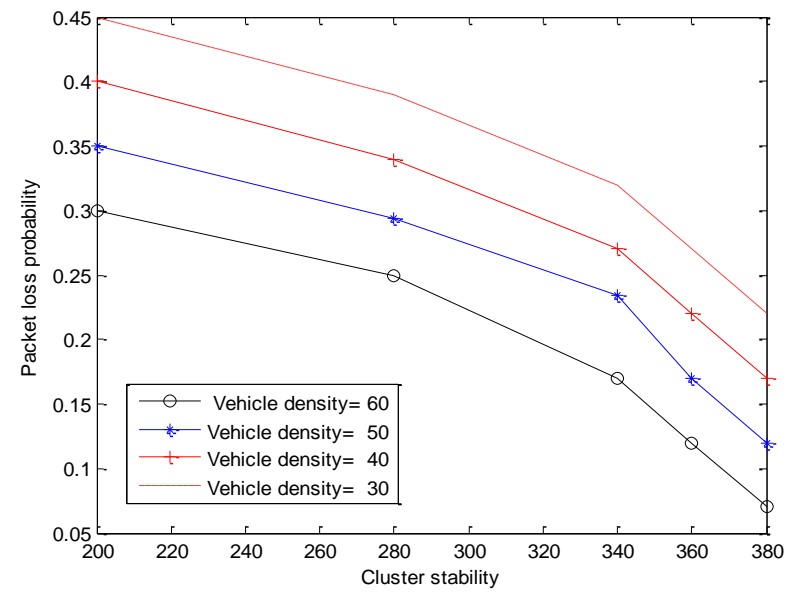

Fig 5. Packet loss probablity under different values of cluster stablity

In Fig. 7, we study VANET performance vs. spectrum cost trade-off under different scenarios of spectrum price and vehicles density. In this experiment, we assign the highest weight to spectrum price as it is the most important factor. Fig. 7 depicts that, as the price increases, packet loss probability increases due to neglecting other important factors for selecting $\mathrm{CH}$ such as signal strength, reachability, location of $\mathrm{CH}, \ldots$, etc. Hence, spectrum cost should be reduced to enhance the performance of VANET communications in terms of packet loss probability, and spectral efficiency, and this will make it cost-effective to deploy and maintain this network. Fig. 8 shows the effect of varying spectrum prices on the lifetime of VANET. From this figure, we observe that the lifetime decreases as the spectrum price increases. This is because the increment of spectrum prices enforces drivers to select the $\mathrm{CH}$ with the lowest prices, and neglects other important heuristics for choosing $\mathrm{CH}$ such as speed of vehicle, the reachability, and the location of the vehicle.

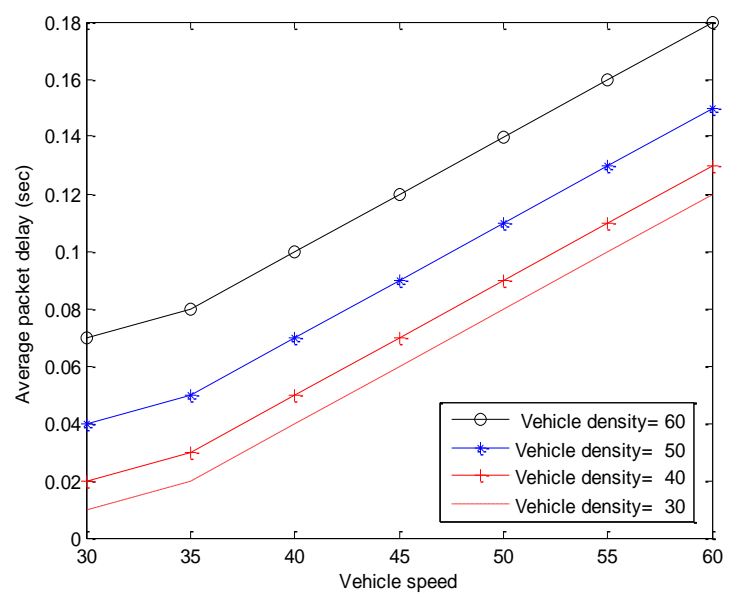

Fig 6. Average packet delay under different valus of vehicle speed 


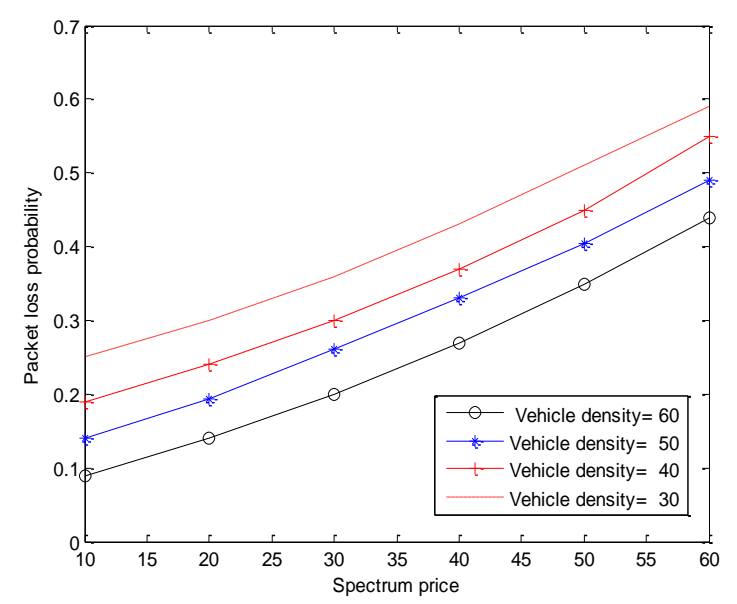

Fig 7. Packet loss probablity under different values of spectrum price

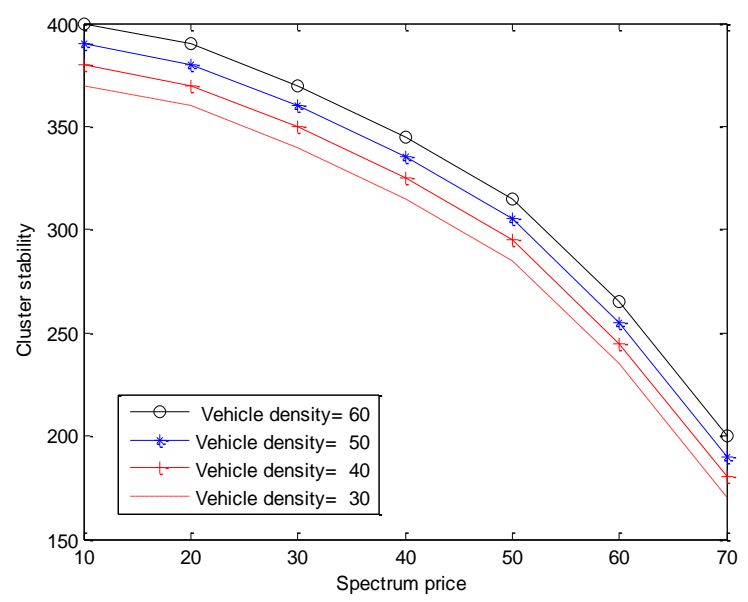

Fig 8. Cluster stablity under different values of spectrum price

\section{CONCLUSION}

This paper emphasizes vehicular network challenges and proposes a novel architecture and incorporated protocols to enhance efficiency and scalability. The $\mathrm{CH}$ selection scheme is a key element for efficient clustering mechanisms in VANET, which influences the stability of the network and overall network performance. The longer the life of the selected $\mathrm{CHs}$ the more the network stability. An innovative fuzzy-based $\mathrm{CH}$ selection scheme is proposed to prolong VANET lifetime. Seven elements were used to select $\mathrm{CH}$ for prolonging the network life time, including speed of vehicle, vehicles' velocity, spectrum price, QoS, number of neighbors, centroid, and signal strength. $\mathrm{CH}$ selection in VANET involves tuning of several conflicting criteria such as spectrum cost, QoS, and reachability degree. These criteria were taken as criteria parameters for the fuzzy control scheme while ranking a set of candidate nodes to select CHs by evaluating a set of criteria. The fuzzy scheme was then tested within different scenarios. Sugeno fuzzy integral is introduced into the $\mathrm{CH}$ selection scheme in order to develop a single criterion for selecting $\mathrm{CHs}$ that can positively impact VANET's lifetime, which is one of the key contributions of this work. The spectrum price effects on VANET stability have been discussed.
Game theory was used in our scheme to model price competition among $\mathrm{CHs}$ for Attracting customers. From the simulation results, we conclude that the proposed scheme effectively prolongs the lifetime of clusters. In our future work, we will focus on studying the impact of these criteria on QoS of VANET, and also explore automated learning and optimization of fuzzy membership functions using state-of-theart deep and reinforcement learning, and novel cognitive control-theoretic elasticity approaches [34]. This could be a concrete step towards optimal $\mathrm{CH}$ selection schemes in VANETs and Intelligent Transportation Systems.

\section{REFERENCES}

[1] S. K. Bhoi and P. M. Khilar, "Vehicular communication: a survey," IET Networks, vol. 3, no. 3, pp. 204-217, 2014.

[2] S. Al-Sultan, M. M. Al-Doori, A. H. Al-Bayatti and H. Zedan, " A comprehensive survey on vehicular ad hoc network," network and computer applications, vol. 37, 380-392, 2014.

[3] R. Hussain, Z. Rezaeifar, H. Oh, "A Paradigm Shift from Vehicular Ad Hoc Networks to VANET-Based Clouds," Wireless Personal Communications, vol. 83, no. 2, pp. 1131-1158, 2015.

[4] A. Alsarhan, A. Y. Al-Dubai, G. Min, A. Y. Zomaya and M. Bsoul, "A New Spectrum Management Scheme for Road Safety in Smart Cities," IEEE Transactions on Intelligent Transportation Systems, vol. 19, no. 11, pp. 3496-3506, 2018

[5] L. A. Zadeh, George J. Klir, Bo Yuan, Fuzzy Sets, Fuzzy Logic, and Fuzzy Systems - Selected Papers by Lotfi A Zadeh. Advances in Fuzzy Systems - Applications and Theory 6, World Scientific 1996, ISBN 978-981-02-2421-9, pp. 1-840.

[6] L. A. Zadeh, "Fuzzy logic - a personal perspective," Fuzzy Sets and Systems, vol. 281, pp. 4-20, 2015.

[7] S. Ucar, S. C. Ergen and O. Ozkasap, "Multihop-Cluster-Based IEEE 802.11p and LTE Hybrid Architecture for VANET Safety Message Dissemination," IEEE Transactions on Vehicular Technology, vol. 65, no. 4, pp. 2621-2636, 2016.

[8] J. Garbiso, A. Diaconescu, M. Coupechoux and B. Leroy, "Auto-adaptive multi-hop clustering for hybrid cellular-vehicular networks," 2017 IEEE 20th Int. Conference on Intelligent Transportation Systems (ITSC), Yokohama, 2017, pp. 1-6.

[9] X. Duan, Y. Liu and X. Wang, "SDN Enabled 5G-VANET: Adaptive Vehicle Clustering and Beamformed Transmission for Aggregated Traffic," IEEE Communications Magazine, vol. 55, no. 7, pp. 120-127, 2017.

[10] V. Goswami, S. K. Verma and V. Singh, "A novel hybrid GA-ACO based clustering algorithm for VANET," 2017 3rd International Conference on Advances in Computing,Communication \& Automation (ICACCA) (Fall), Dehradun, India, 2017, pp. 1-6.

[11] M. Azizian, S. Cherkaoui and A. S. Hafid, "A distributed D-hop cluster formation for VANET," 2016 IEEE Wireless Communications and Networking Conference, Doha, 2016, pp. 1-6.

[12] M. Ren, L. Khoukhi, H. Labiod, J. Zhang, and V. Véque, "Amobility-based scheme for dynamic clustering in vehicular ad-hoc networks (VANETs)," Vehicular $\begin{array}{lll}\text { Communications, } & 2016 . & \text { [Online]. }\end{array}$ Available:http://dx.doi.org/10.1016/j.vehcom.2016.12.003.

[13] A. Malathi, and N. Sreenath, " An Efficient Clustering Algorithm for Vanet," International Journal of Applied Engineering Research, vlo. 12, no. 9, pp. 20002005, 2017.

[14] Shea, C., Hassanabadi, B., \& Valaee, S. (2009, November). Mobility-based clustering in VANETs using affinity propagation. In Global telecommunications conference, 2009. GLOBECOM 2009. IEEE (pp. 1-6). IEEE.

[15] H. Wang, R. P. Liu, W. Ni, W. Chen and I. B. Collings, "VANET Modeling and Clustering Design Under Practical Traffic, Channel and Mobility Conditions," IEEE Transactions on Communications, vol. 63, no. 3, pp. 870-881, 2015.

[16] Y. Huo, W. Dong, J. Qian, and T. Jing, "Coalition game-based secure and effective clustering communication in vehicular cyber-physical system (VCPS)," Sensors, vol. 17, no. 3, p. E475, 2017.

[17] Z. Y. Rawashdeh and S. Mahmud, "A novel algorithm to form stable clusters in vehicular ad hoc networks on highways," Eurasip journal on Wireless communications and networking, vol. 2012, no. 1, p. 15, 2012.

[18] H. R. Arkian, R. E. Atani, A. Pourkhalili, and S. Kamali, "Cluster-based traffic information generalization in vehicular ad-hoc networks," Vehicular communications, vol. 1, no. 14, pp. 197-207, 2014.

[19] Y. Chen, M. Fang, S. Shi, W. Guo and X. Zheng, "Distributed multi-hop clustering algoritm for VANETs based on neighborhood follow," journal on wireless communications and networking, vol. 2015, no. 98, pp. 1-12, 2015.

[20] Ren, M., Zhang, J., Khoukhi, L., Labiod, H., \& Vèque, V. (2017). A Unified Framework of Clustering Approach in Vehicular Ad Hoc Networks. IEEE Transactions on Intelligent Transportation Systems. 
[21] A. Benslimane, T. Taleb and R. Sivaraj, "Dynamic Clustering-Based Adaptive Mobile Gateway Management in Integrated VANET - 3G Heterogeneous Wireless Networks," IEEE Journal on Selected Areas in Communications, vol. 29, no. 3, pp. 559-570, 2011.

[22] Kannekanti, S. Y., Nunna, G. S., Bobba, V. K. R., Yadama, A. K., \& Elleithy, A. (2017, October). An efficient clustering scheme in vehicular Ad-Hoc networks. In Ubiquitous Computing, Electronics and Mobile Communication Conference (UEMCON), 2017 IEEE 8th Annual (pp. 282-287).

[23] H. Su and X. Zhang, "Clustering-Based Multichannel MAC Protocols for QoS Provisionings Over Vehicular Ad Hoc Networks," IEEE Transactions on Vehicular Technology, vol. 56, no. 6, pp. 3309-3323, 2007.

[24] A. Abuashour and M. Kadoch, "Performance Improvement of Cluster-Based Routing Protocol in VANET," in IEEE Access, vol. 5, pp. 15354-15371, 2017.

[25] Chenhao Shi, Yi Zhou, Wei Li, Huanhuan Li, Ning Lu, Nan Cheng, Tingting Yang"A Centralized Clustering Based Hybrid Vehicular Networking Architecture for Safety Data Delivery," GLOBECOM 2017 - 2017 IEEE Global Communications Conference, Singapore, 2017, pp. 1-6.

[26] X. Li, T. Song, Y. Zhang, G. Chen and J. Hu, "A Hybrid Cooperative Spectrum Sensing Scheme Based on Spatial-Temporal Correlation for CR-VANET," 2018 IEEE 87th Vehicular Technology Conference (VTC Spring), Porto, 2018, pp. 1-6

[27] M. F. Khan, E. A. Felemban, S. Qaisar and S. Ali, "Performance Analysis on Packet Delivery Ratio and End-to-End Delay of Different Network Topologies in Wireless Sensor Networks (WSNs)," 2013 IEEE 9th International Conference on Mobile Adhoc and Sensor Networks, Dalian, 2013, pp. 324-329.

[28] Pedro A. Forero, Alfonso Cano, and Georgios B. Giannakis, "Distributed Clustering Using Wireless Sensor Networks, " IEEE Journal Of Selected Topics In Signal Processing, vol. 5, no. 4, 2011.

[29] Rappaport, Theodore S. Wireless Communications: Principles and Practice. Upper Saddle River, N.J.: Prentice Hall PTR, 2002.

[30] Sugeno, M. Theory of fuzzy integrals and its applications. Ph.D. Dissertation, Tokyo Institute of Technology, Tokyo, Japan. 1974.

[31] G. H. Tzeng, Y. P. OuYang, C. T. Lin, and C. B. Chen, " Hierarchical MADM with fuzzy integral for evaluating enterprise intranet web sites," Information Sciences, vol. 169, no. 3, pp. 409-426. 2005.

[32]L. Jin, R. Mesiar and G. Qian, "Weighting Models to Generate Weights and Capacities in Multicriteria Group Decision Making," in IEEE Transactions on Fuzzy Systems, vol. 26, no. 4, pp. 2225-2236, Aug. 2018.

[33] M. Mahmud; M.S. Kaiser; A. Hussain \& S. Vassanelli; "Applications of Deep Learning and Reinforcement Learning to Biological Data", IEEE Transactions in Neural Networks and Learning Systems, vo. 29, no.6, pp.2063-2079, 2018

[34]A. Ullah, J. Li, A. Hussain \& Y. Shen; "Genetic optimization of fuzzy membership functions for cloud resource provisioning," 2016 IEEE Symposium Series on Computational Intelligence (SSCI), Athens, 2016,pp.1-8. (doi: 10.1109/SSCI.2016.7850088)

[35] A. Mas-Colell, M. Whinston, J. Green, "Microeconomic Theory”, Oxford University Press, 1995

[36] M. J. Osborne, and C. Pitchik, "Price Competition in a Capacity-Constrained Duopoly”, Economic Theory, vol. 38, no. 2, pp. 238-260, 1986.

[37] E. Marchi, "Equilibrium points of rational n-person games," Journal of Mathematical Analysis and Appilicationas, vol. 54, no. 1, 1979.

[38] R. Nessah and G. Tian, "On the existence of nash equilibrium

in discontinuous games," Economic Theory, vol. 61, no. 2, pp. 515-540, 2016.

[39] K. Zhang, Y. Mao, S. Leng, Y. He, and Y. Zhang, “Mobile-edge computing for vehicular networks: A promising network paradigm with predictive offloading," IEEE Veh. Technol. Mag., vol. 12, no. 2, pp. 36_44, Jun. 2017.

[40] N. Cordeschi, D. Amendola, and E. Baccarelli, "Reliable adaptive resource management for cognitive cloud vehicular networks," IEEE Trans. Veh. Technol., vol. 64, no. 6, pp. 2528_2537, Jun. 2015.

[41] G. el M. Zhioua, H. Labiod, N. Tabbane, and S. Tabbane, “VANET inherent capacity for offloading wireless cellular infrastructure: An analytical study," in Proc. 6th IEEE Int. Conf. New Technol., Mobility Secur. (NTMS), 2014, pp. 1-5.

[42] G. el M. Zhioua, J. Zhang, H. Labiod, N. Tabbane, and S. Tabbane, "'VOPP: A VANET offloading potential prediction model," in Proc. IEEE Wireless Commun. Netw. Conf. (WCNC), Apr. 2014, pp. 2408_2413.

[43] G. El M. Zhioua, H. Labiod, N. Tabbane, and S. Tabbane, “Cellular content download through a vehicular network: I2V link estimation," in Proc. 81th IEEE Veh. Technol. Conf. (VTC Spring), May 2015, pp. 1-6.

[44] N. Abbas, H. Hajj, S. Sharafeddine and Z. Dawy, "Traffic Offloading With Channel Allocation in Cache-Enabled Ultra-Dense Wireless Networks," in IEEE Transactions on Vehicular Technology, vol. 67, no. 9, pp. 8723-8737, Sept. 2018.

[45] H. Guo, J. Liu and J. Zhang, "Computation Offloading for Multi-Access Mobile Edge Computing in Ultra-Dense Networks," in IEEE Communications Magazine, vol. 56, no. 8, pp. 14-19, August 2018.

[46] C. Andre, S. Susana, and S. Peter, "On the Connection Availability between Relay Nodes in a VANET," IEEE Globecom, pp. 181-185, 2010.

[47] L. Liu, C. Chen, T. Qiu,M. Zhang, S. Li, and B. Zhou, "A data dissemination scheme based on clustering and probabilistic broadcasting in VANETs," Vehicular Communications, vol. 13, pp. 78-88, 2018.

[48] B. Subba, S. Biswas, and S. Karmakar, "A game theory based multi layered intrusion detection framework for wireless sensor networks," International Journal of Wireless Information Networks, vol. 25, no. 4, pp. 399-421, 2018.
[49] C. BrijilalRuban and B. Paramasivan, "Cluster-based secure communication and certificate revocation scheme for VANET," The Computer Journal, vol. 62, no. 2, pp. 263-275, 2019.

[50] A. Sahoo, S. K. Swain, B. K. Pattanayak, and M. N. Mohanty, "An optimized cluster based routing technique in vanet for next generation network," in Information Systems Design and Intelligent Applications, S. C. Satapathy, J. K. Mandal, S. K. Udgata, and V. Bhateja, Eds., pp. 667-675, Springer, New Delhi, India, 2016.

[51] V. Sethi and N. Chand, "A destination based routing protocol for context based clusters in VANET," Communications and Network, vol. 09, no. 03, pp. 179-191, 2017.

[52] A. Shahen Shah, H. Ilhan, and U. Tureli, "CB-MAC: a novel cluster-based MAC protocol for VANETs," IET Intelligent Transport Systems, 2018.

[53] C. Cooper, D. Franklin, M. Ros, F. Safaei, and M. Abolhasan, "A comparative survey of VANET clustering techniques," IEEE Communications Surveys \& Tutorials, vol. 19, no. 1, pp. 657-681, 2017.

[54] K. D. Singh, P. Rawat, and J. M. Bonnin, "Cognitive radio for vehicularad hoc networks (CR-VANETs): Approaches and challenges,''EURASIPJ. Wireless Commun. Netw., vol. 14, no. 1, pp. 1-22, 2014.

[55] W. Sun and J. Liu, "2-to- \$M\$ Coordinated Multipoint-Based Uplink Transmission in Ultra-Dense Cellular Networks," in IEEE Transactions on Wireless Communications, vol. 17, no. 12, pp, Oct. 2018.

[56] W. Sun, J. Liu, Y. Yue and H. Zhang, "Double Auction-Based Resource Allocation for Mobile Edge Computing in Industrial Internet of Things," in IEEE Transactions on Industrial Informatics, vol. 14, no. 10, pp. 4692-4701, Oct. 2018.

[57] X. Wang, Y. Han, C. Wang, Q. Zhao, X. Chen, and M. Chen,"In-edge AI: Intelligentizing mobile edge computing, cachingand communication by federated learning,"ArXiv preprint,vol. abs/1809.07857, Sept. 2018.

[58] H. Guo, J. Liu, J. Zhang, W. Sun and N. Kato, "Mobile-Edge Computation Offloading for Ultradense IoT Networks," in IEEE Internet of Things Journal, vol. 5, no. 6, pp. 4977-4988, Dec. 2018.

[59] H. Guo and J. Liu, "Collaborative Computation Offloading for Multiaccess Edge Computing Over Fiber-Wireless Networks," in IEEE Transactions on Vehicular Technology, vol. 67, no. 5, pp. 4514-4526, May 2018.

[60] Y. N. Shnaiwer, S. Sorour, P. Sadeghi, N. Aboutorab and T. Y. Al-Naffouri, "Network-Coded Macrocell Offloading in Femtocaching-Assisted Cellular Networks," in IEEE Transactions on Vehicular Technology, vol. 67, no. 3, pp. 26442659, March 2018.

[61] N. Abbas, H. Hajj, S. Sharafeddine and Z. Dawy, "Traffic Offloading With Channel Allocation in Cache-Enabled Ultra-Dense Wireless Networks," in IEEE Transactions on Vehicular Technology, vol. 67, no. 9, pp. 8723-8737, Sept. 2018.

[62] H. Guo, J. Zhang and J. Liu, "FiWi-Enhanced Vehicular Edge Computing Networks: Collaborative Task Offloading," in IEEE Vehicular Technology Magazine, vol. 14, no. 1, pp. 45-53, March 2019.

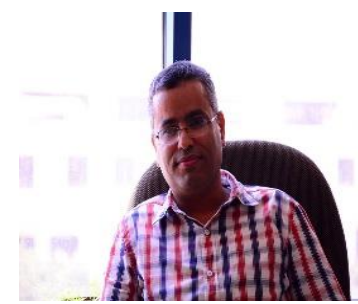

Ayoub Alsarhan received his Ph.D. degree in Electrical and Computer Engineering from Concordia University, Canada in 2011, his M.Sc. Degree in Computer Science from Al-Bayt University, Jordan in 2001, and B.E. degree in Computer Science from the Yarmouk University, Jordan in 1997. He is currently an Associate Professor at the Computer Information System Department of the Hashemite University, Zarqa, Jordan. His research interests include Cognitive Networks, Parallel Processing, Cloud Computing, Machine Learning, and Real Time Multimedia Communication over Internet.

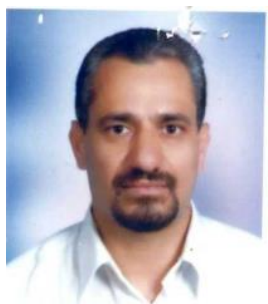

Yousef Kilani received his $\mathrm{PhD}$ in the Computer Science from the University of Kebangsaan Malaysia, in 2007, MSc in Computer Science from the University of Science Malaysia, in 1997, and bachelor's degree in Computer Science from Yarmouk University, Jordan, in 1994. He is currently an Associate Professor at the Department of Computer Information Systems at Hashemite University, Zarqa, Jordan. His research interests include artificial intelligence, web intelligence, machine learning and real time multimedia communication over the internet. 


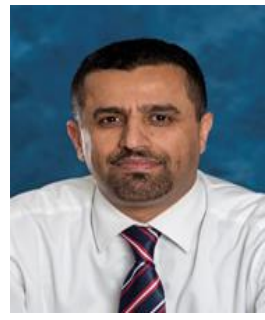

Ahmed Y Al-Dubai received his Ph.D. in Computing from University of Glasgow in 2004. In 2004, he joined the University of West London and then in 2005 he joined Edinburgh Napier University where he became a Professor and the Program Leader of the Postgraduate Research Degrees of the School of Computing. He is the Head of the Networks Research Group. He has been working on the area of group communication algorithms, smart spaces and high-performance networks. He published in world leading journals including IEEE Transaction and in prestigious international conferences. He has been the recipient of several academic awards and recognitions and a member of several editorial boards of scholarly journals. He served as Guest Editor for over 20 special issues in scholarly journals and Chaired and Co-chaired over 30 International conferences/workshops. Prof. Al-Dubai is a Fellow of the British Higher Academy and a Senior Member of the IEEE.

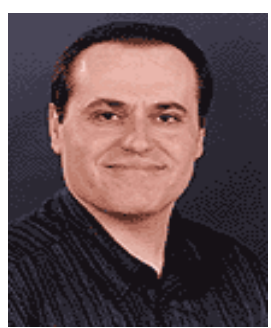

Albert Y. Zomaya is the Chair Professor of High Performance Computing \& Networking in the School of Computer Science, University of Sydney, and he also serves as the Director of the Centre for Distributed and High Performance Computing. Professor Zomaya published more than 600 scientific papers and articles and is author, co-author or editor of more than 20 books. He is the Founding Editor in Chief of the IEEE Transactions on Sustainable Computing and serves as an associate editor for more than 20 leading journals. Professor Zomaya served as an Editor in Chief for the IEEE Transactions on Computers (2011-2014). Professor Zomaya is the recipient of the IEEE Technical Committee on Parallel Processing Outstanding Service Award (2011), the IEEE Technical Committee on Scalable Computing Medal for Excellence in Scalable Computing (2011), and the IEEE Computer Society Technical Achievement Award (2014). He is a Chartered Engineer, a Fellow of AAAS, IEEE, IET, and an Elected Member of Academia Europaea. Professor Zomaya's research interests are in the areas of parallel and distributed computing and complex systems.

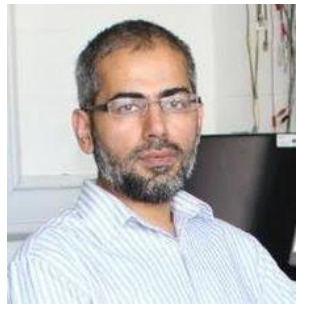

Amir Hussain received his B.Eng (highest 1st Class Honours with distinction) and Ph.D degrees, from the University of Strathclyde, Glasgow, U.K., in 1992 and 1997, respectively. Following postdoctoral and academic positions held at the West of Scotland (1996-98), Dundee (1998-2000) and Stirling Universities (2000-18) respectively, he is currently Professor and founding Head of the Cognitive Big Data and Cybersecurity (CogBiD) Research Lab at Edinburgh Napier University, U.K. He has (co)authored more than 350 papers, including over a dozen books and around 140 journal papers. He is founding Editor-in-Chief of two leading journals: Cognitive Computation (Springer Nature), and BMC Big Data Analytics (BioMed Central), and of the Springer Book Series on Socio-Affective Computing, and Cognitive Computation Trends. He is Associate Editor for a number of prestigious journals, including: Information Fusion (Elsevier), AI Review (Springer), the IEEE Transactions on Neural Networks and Learning Systems, the IEEE Computational Intelligence Magazine, and the IEEE Transactions on Emerging Topics in Computational Intelligence. Amongst other distinguished roles, he is General Chair for IEEE WCCI 2020 (the world's largest technical event in Computational Intelligence). 